\title{
Wirkungen des pleistozänen kaltzeitlichen Klimas, insbeson- dere des Bodenfrostes, in den Sedimenten des östlichen Ostfalen (Eiskeile, Löß-Flugsand, asymmetrische Täler)
}

\author{
KARL-UlRICH BROSCHE*)
}

\begin{abstract}
Key-words: Elsterian, Saalian and Weichselian periglacial structures, epigenetic and syngenetic ice-wedge casts, Northern loess border and their sediments, climatic asymmetric valleys, Eastern Lower Saxony "Ostfalen"
\end{abstract}

Kurzfassung: Im zweiten Teil des Beitrags wird eine reiche Dokumentation von Abbildungen vorgenommen, um das Inventar von Eiskeilen im östlichen Teil von Niedersachsen (Ostfalen) zu analysieren. Eiskeile sind die einzigen Periglazialerscheinungen, die zweifelsfrei einen ehemaligen Dauerfrostboden repräsentieren. Nach einer kurzen Einleitung werden im 2. Kapitel zwei saalezeitliche oder elsterzeitliche Eiskeilnetze dokumentiert, welche in Ahlum (östlich von Wolfenbüttel) bis in eine Tiefe von $6 \mathrm{~m}$ herabreichen und welche sehr gut datiert werden können durch ihr Füllmaterial und die Deckschichten. In Barmke (nordwestl. von Helmstedt, Nr. 12, Abb. 1) wurden im Rahmen eines größeren Eiskeilnetzes normale Eiskeile und vorzeitliche „Eisgänge“ (ice-vains) angetroffen (Abb. 3), welche ebenfalls eine saalezeitliches oder elsterzeitliches Alter besitzen. Die Eiskeile mit einer Länge von 2,0 bis 2,5 $\mathrm{m}$, die in einer zusammenhängenden Phase gebildet wurden, sind alle von weichselzeitlichem Alter (Kap. 3). In manchen Exemplaren liegen polygenetische Eiskeile vor, die entweder während der Saale- (oder der Elster-Zeit) oder - in anderen Fällen - während der Saale- und Weichselzeit wuchsen (Kap. 4). - In Kapitel 5 werden einige „Kleinkeile“ und Kissenböden mit kleinen syngenetischen Frostspalten präsentiert, deren Bildung nicht in eine Permafrostperiode fällt, sondern die während einer weniger kalten stadialen oder interstadialen Phase während der Weichselkaltzeit gebildet sein müssen (Abb. 15 und 16). Eine Abfolge von gelbem Löß in Abwechslung mit weißen Sanden, die aus dem Eozän der Helmstedter Tertiärmulde stammen, stellt eine Ausnahme dar (vgl. GeErt \& HaGedorN 1996). - Nur zwei klimatisch bedingte asymmetrische Täler, beide etwa in Süd-Nord-Richtung verlaufend, wurden gefunden. Sie wurden in Quartärsedimenten entwickelt oder in Sanden des Tertiärs (Miozäns, Oligozäns, Eozäns). Nur einige Eiskeile sind in Mergeln des Mittleren Keupers ausgebildet. - Syngenetische Kryoturbationsformen und Eiskeilpseudomorphosen wurden nur in der Oker-Mittelterrasse nördl. Isingerode angetroffen. - An klimatischen Schlüssen kann für die kälteste Zeit der Weichsel- und Saale-Kaltzeit geschlossen werden, daß die Temperaturdepression (im Jahresdurchschnitt) mindestens 14 bis $15^{\circ}$ betragen hat.

*) Anschrift des Verfassers: Prof. Dr. K.-U. Brosche, Institut für Geographische Wissenschaften der FU Berlin, Fachrichtung Physische Geographie, Grunewaldstr. 35, 12165 Berlin

\author{
[Action of pleistocene cold climate, especially \\ of soil frost in the sediments of eastern Ostfalen - \\ region of Hannover - Wolfsburg - Helmstedt - \\ Bad Harzburg - Bad Salzgitter - Hannover]
} "Wirkungen des ..." a various documentation of pictures is presented to analyse the ice-wedge casts of the eastern part of Lower Saxony (Ostfalen). These structures are the only periglacial phenomena, which represent doublessly a former permafrost soil. After a short introduction, in chapter 2 Saalian or probably older nets of ice-wedge casts are documentated, which reach in a depth of $6 \mathrm{~m}$ in Ahlum (east of Wolfenbüttel) and which can be dated very well using their content of sediments and their cover sediments. In Barmke (nr. 12, fig. 1) there were found normal ice-wedge casts and former ice-vains and "Eisgänge" (fig.2), which posess a Saalian or older age. - The ice-wedge casts with a length of 2,0 to $2,5 \mathrm{~m}$, which were formed in one continuing phase are all of Weichselian age. - In some exemples polygenetic ice-wedge casts could be found, which grew either during the Saalian (or the Elsterian) or - in other examples - during the Saalian and Weichselian time. - In chapter 5 some „Kleinkeile“ and „Kissenböden“ with little syngenetic frost fissures are presented, which are not due to a permafrost soil, but are formed by a frost soil during a stadial or interstadial phase within the Weichselian (fig. 14 and 15). - A sequence of a yellow loess in alternation with sand layers from the Eocene formation, which was a great exception, was presented from the northern loess border (fig. 16). - Only two climatic asymmetric valleys could be found, which were developed since the Saalian ice left our landscape. - All described forms were developed in sediments of the Quaternary or in sands and clay of the Tertiary. Only some ice-wedge casts were formed in marls and clay of the Keuper. - Syngenetic cryoturbation forms and ice-wedge casts could only be found in the Oker-Mittelterrasse (fig. 18). - Climatic conclusions for the coldest times of the Weichselian and Saalian time were made: at least a temperature depression of 14 to $15^{\circ}$ could be found.

\section{Einleitung: Gegenstand der Untersuchung}

Dieser Beitrag stellt die Fortsetzung des gleichnamigen Aufsatzes aus dem Heft 46 von Eiszeitalter und Gegenwart dar, in dem hauptsächlich glazigene 
- Eiskeilpseudomorphose (weichselzeitlich)

ice-wedge cast, weichselian

Eiskeilpseudomorphose (älter als weichselzeitlich

$\nabla$ oder mehrphasig)

ice-wedge cast, older than weichselian or polygenetic

Kryturbationsformen, älter als

- weichselzeitlich angelegt

proved periglacial involutions

(pocket soil etc.)

W Wechsel von Sand- und Lößschichten an der

sekundär-asymmetrisches Ta

(ab Rehburger Stadium geformt)

Stauchfalten in saalezeitlichen oder

$\approx$ älteren Schichten ( $z$.T. unter Einbeziehung

älterer Sedimente)

tektonischer "Stich"

Al atektonische Faltung

\section{.... Steinsohle}

$\approx$ mehrphasige Solifluktionsschuttdecke
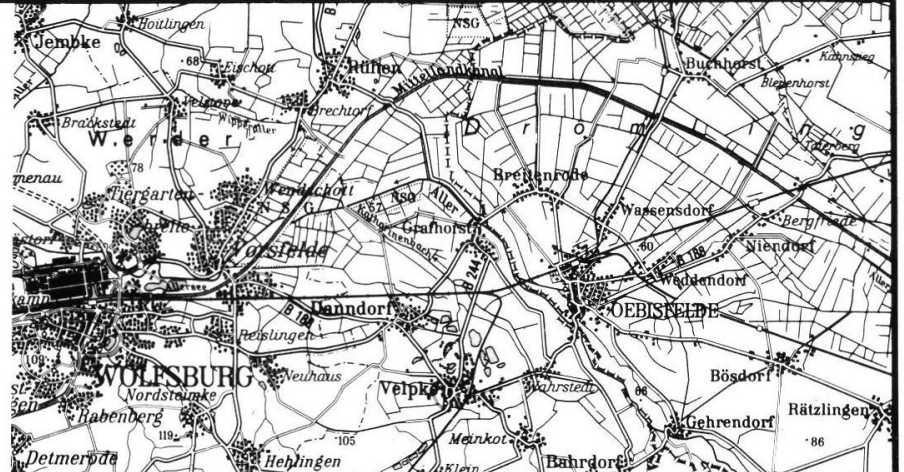
Detmerpde

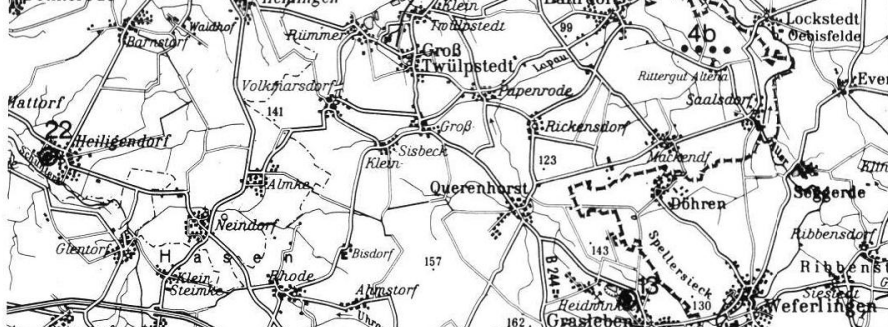
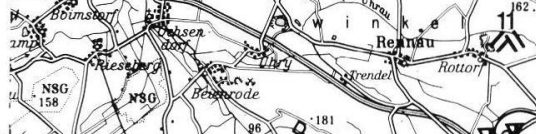

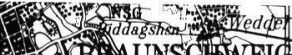

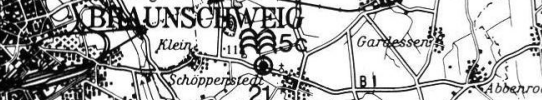

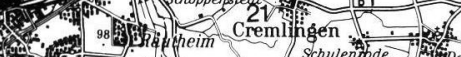

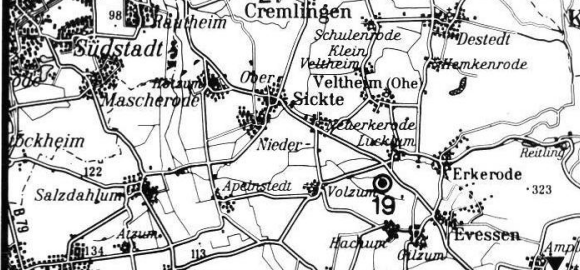

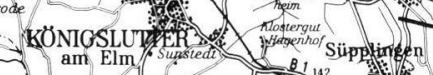

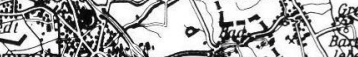
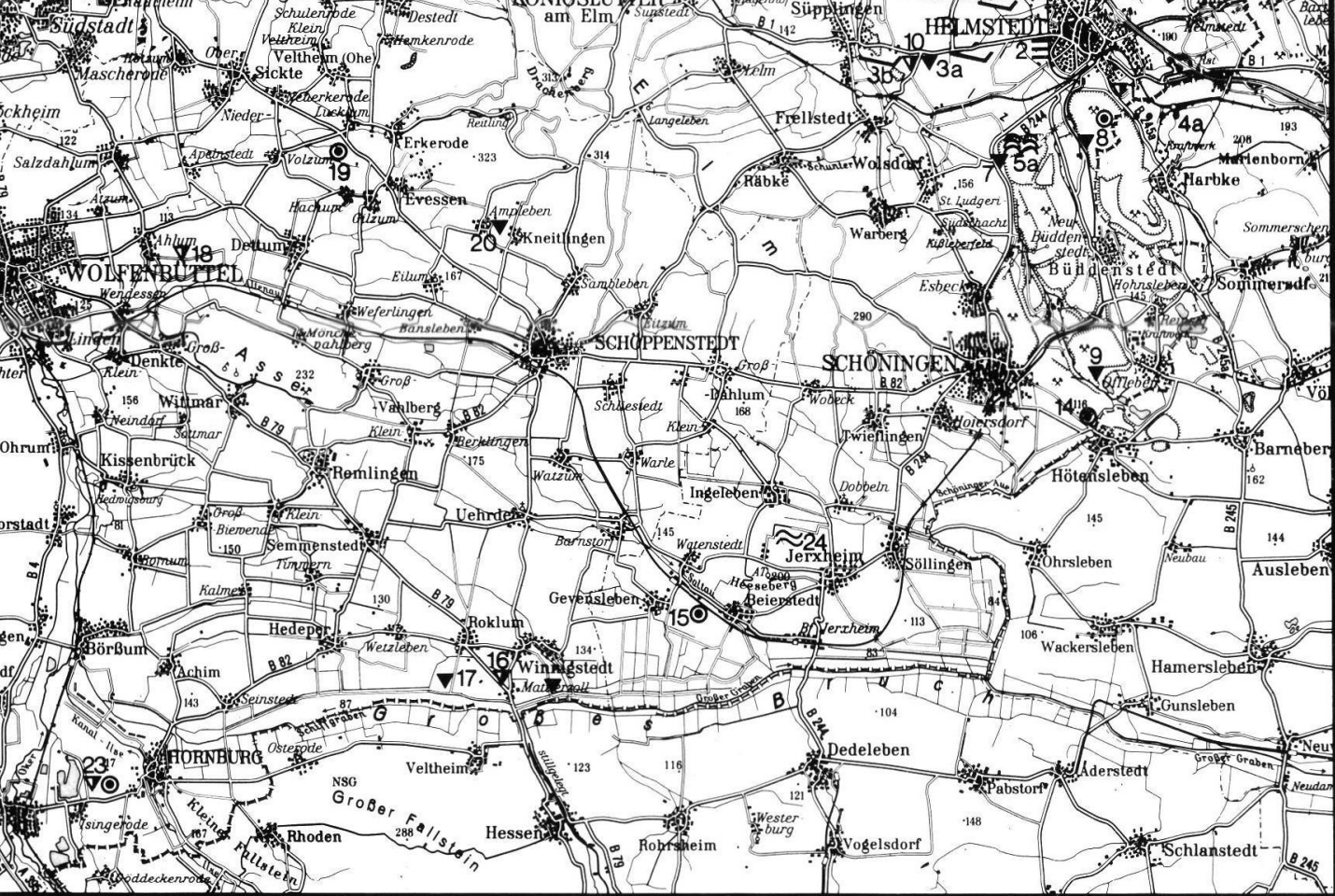

Abb. 1 Karte der Periglazialerscheinungen in Ostfalen

Fig. 1 Map of periglacial structures in Ostfalen 


\section{Zusammenstellung der wichtigsten Periglazialerscheinungen in Ostfalen, zugleich Erläuterung zu Abbildung 1}

1. Weichselzeitliche oder ältere Eiskeile südlich des Ludgeri-Kreuzes bei Helmstedt (saalezeitliche oder elsterzeitliche, rotbraun verwitterte Sande als Füllmaterial in hangab verschleppten Eiskeilen und Frostspalten im Keupermergel), Länge 0,8-1,0 m, Breite 5-10 cm; außerdem Kryoturbationsformen, u. a. Wickelboden - vgl. BROsche (1964) - gleichen Alters.

2. Wechselfolge von weichselzeitlichen Lößschichten und eozänen Sandschichten unter einem weichselzeitlichem gelbem Löß in einer Baugrube in der Heinrich-Kremp-Straße im Helmstedter Stadtgebiet (Abb. 17). Nähe der nördlichen Lößgrenze.

3a. Sekundär-asymmetrisches Tal westl. der Lübbensteine, westl. Helmstedt in eozänen Sanden und quartären Deckschichten.

3b. dto. im S-N ziehenden Schuntertal nördl. Frellstedt (Abb. 18). Osthang zwischen Frellstedt und Süpplingenburg $20-25^{\circ}$ geneigt.

4a. Steinsohle an Grenze Weichsellöß zu saalezeitlicher Grundmoräne in ehemaliger Tongrube Stegemann (südl. Helmstedt an der ehemaligen Feldscheune - heute Tagebau Helmstedt der BKB Helmstedt - Abb. 11 bei Brosche 1964).

5a. Stauchfalten (z. T. mit Spezialfältelung - Abb. 7 bei Brosche 1996) an der N- und E-Wand des ehemaligen Tagebaus Treue der BKB (Braunschweigische Kohlenbergwerke Helmstedt).

5b. dto. in der ehemaligen Ziegeleigrube Lehrmann am ehemaligen Bahnhof Emmerstedt (Abb. 6 bei Brosche 1996).

5c. dto. im Endmoränenzug des Rehburger Stadiums (Saaleeiszeit) südl. Weddel, Abb 13 bei Brosche (1996).

6. Sonderform einer möglichen Kryoturbationserscheinung in der ehemaligen Ziegeleigrube Stegemann am Weinberg nördl. Helmstedt (südl. der ehemaligen Abdeckerei)- vgl. Abb. 8 bei Brosche (1996).

7. Eiskeilpseudomorphosen an $\mathrm{N}$-Wand des ehemaligen Tagebaus Treue der BKB Helmstedt. Länge 1,8 - 2,0 m, Breite 0,3 - 0,4 m, weichselzeitliche Formen - Abb. 11.

8. dto. an S-Wand des Tagebaus Helmstedt - Abb. 12 in diesem Beitrag.

9. dto. an E-Wand des Tagebaus Alversdorf (vgl. Brosche \& Walter 1991, Abb. 9 u. 10, Foto 3-5). Weichelzeitliche Eiskeile in drei Eiskeilstockwerken.

10. Weichselzeitliche Eiskeile mit 1,8-2,0 m Länge und 0,5 m Breite; saalezeitliche Eiskeile in saalezeitlichen glazifluvialen Sedimenten am Nordschacht, $5 \mathrm{~km}$ westl. Helmstedt in der Eversschen Kiesgrube: mehrphasige $5 \mathrm{~m}$ lange Eiskeile - Abb. 9,13 in diesem Beitrag.

11. Tektonischer Stich i. S. KerlнаскS (1931), auch atektonische Faltung genannt, in ehemaliger Eisensandsteingrube nördl. Rottorf im Liassandstein nordwestl. Helmstedt - Abb. 3 bei Brosche (1996).

12. Saalezeitliches oder elsterzeitliches Eiskeilnetz mit Eisadern bzw. Eisgängen von $2 \mathrm{~m}$ Tiefe in der ehemaligen Gemeindemergelgrube Barmke am Krähenberg östl. Barmke (Mergel des Mittleren Keupers). Abb. 2-5 in diesem Beitrag.

13. Riesen-Wannen- und Kesselböden in der ehemaligen Ziegeleigrube Grasleben (am nördl. Ortsrand von Grasleben). Wahrscheinlich ab der Elster- oder Saale-Eiszeit gebildet. Abb. 16 bei Brosche (1996).

14. Ehemalige Kiesgrube westl. Hötensleben bei Schöningen, Kryoturbationsformen mit Taschen- und Spitzenböden in wahrscheinlich saalezeitlichen Sedimenten unter weichselzeitlichem Löß. Abb. 9, 10 bei BrosCHE (1996).

15. „Girlandenboden“ in der Gemeindekiesgrube westl. Beierstedt. Wahrscheinlich in saalezeitlichen Sedimenten und wahrscheinlich von saalezeitlichem Alter. Abb. 11 bei BROSCHE (1996).

16. Mehrphasiger Eiskeil von 5,5 m Länge in der Gemeindekiesgrube Winnigstedt, südl. Schöppenstedt. Saalezeitlich (im unteren Teil) und weichselzeitlich im oberen Teil geformt. Abb. 14 in diesem Beitrag. Daneben ein $1 \mathrm{~m}$ langer plumper weichseleiszeitlicher Eiskeil mit weichselzeitlicher Lößfüllung.

17. Weichselzeitliche Eiskeile in der ehemaligen Ziegeleigrube Roklum an der B 79 Wolfenbüttel - Halberstedt, von weichselzeitlichem Löß gefüllt. Plumpe Formen wie in Abb. 9 sichtbar und Sonderform (Abb. 10 in diesem Beitrag). Die weiselzeitliche Lößfüllung greift in Neokomton hinein.

18. Eiskeilnetz mit $6 \mathrm{~m}$ langen Eiskeilen in der ehemaligen Gemeindekiesgrube Ahlum am östl. Ortsrand von Ahlum (östl. Wolfenbüttel). Abb. 6, 7 in diesem Beitrag.

19. Kryoturbationsformen („Girlandenboden“) in der ehemaligen Kiesgrube Lucklum (westl. des Elms). Abb. 12 bei BROSCHE (1996).

20. Eiskeile in der ehemaligen Schotter- und Lößgrube nordöstl. Ampleben bei Schöppenstedt (vgl. Brosche \& WALTHER 1991, S. 35, Abb. 13). Weichselzeitlicher Löß als Eiskeilfüllmaterial in saalezeitlichen glazifluvialen Sedimenten.

21. Kryoturbationsformen, möglicherweise mehrphasige Bildungen, in glazifluvialen, gestauchten Sedimenten des Endmoränenzuges des Rehburger Stadiums südl. Weddel. Abb. 13 bei Brosche (1996).

22. Taschenböden in der ehemaligen Gemeindekiesgrube am ehemaligen westl. Ortsrand von Heiligendorf. Abb. 14 bei BROSCHE (1996).

23. Syngenetische saalezeitliche Eiskeile und syngenetische saalezeitliche Kryoturbationsformen (Würge- und Taschenböden) in der Oker-Mittelterrasse nördl. Isingerode (südl. Wolfenbüttel). Eiskeile z. Z. nicht mehr aufgeschlossen; Kryoturbationsformen mit Dimensionen von 1,0 bis 1,2 $\mathrm{m}$ im Ostteil der Kiesgrube noch gut aufgeschlossen. Abb. 18 in diesem Beitrag.

24. Elster- oder saalezeitliche und weichselzeitliche Solifluktionsschuttdecke nördl. des Heeseberges westl. von Jerxheim. Abb. 5 bei BROSCHE (1996).

\section{Außerhalb der Kartendarstellung:}

25. Saalezeitliche oder elster- und saalezeitliche Kryoturbationsformen (eventuell von syngenetischem Charakter) in den Oberterrassensedimenten des Weddebaches nördl. Weddingen (nördl. des Harliberges nördl. Goslar). Abb. 15 bei Brosche (1996).

26. Hakenschlagen in einer wahrscheinlich mehrphasig entstandenen Solifluktionsschuttdecke des Mittleren Buntsandsteins auf dem Friedhof von Salzgitter-Bad am Hamberg. Abb. 4 bei Brosche (1996).

27. Gelivationshorizont mit „Frostkesseln“ im Doggersandstein bei Harenberg (westl von Hannover). Abb. 2 bei BROSCHE (1996). 


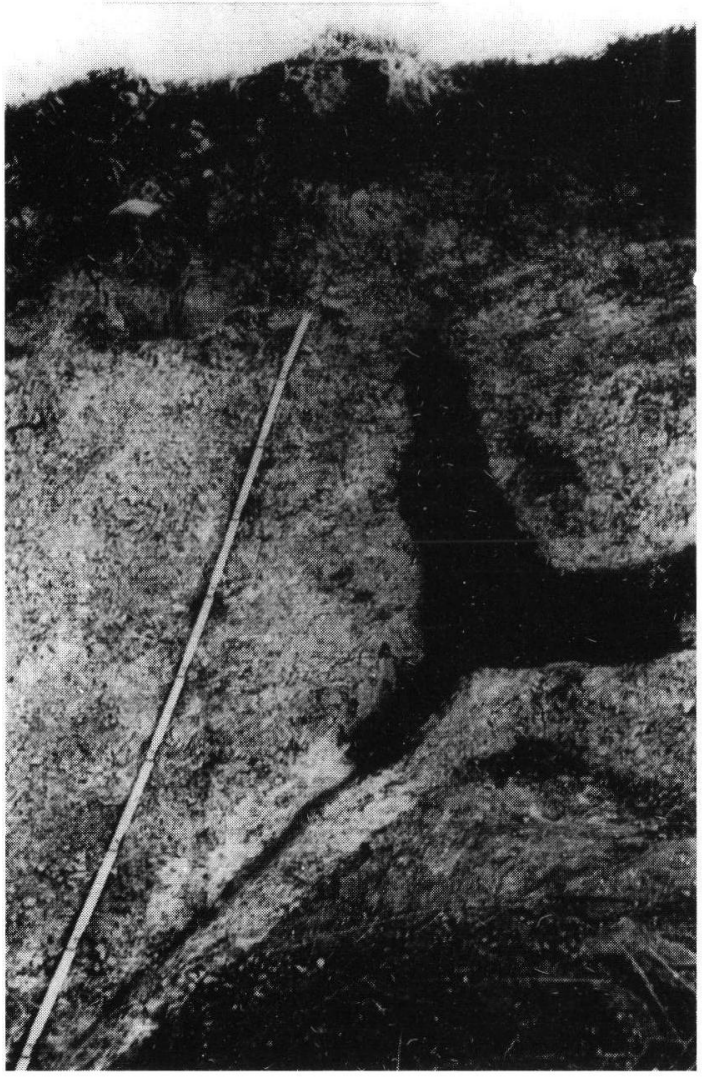

Abb. 2: Ehemalige Mergelgrube am Krähenberg östl. Barmke (nordwestl. Helmstedt). Ausschnitt aus der ein Eiskeilnetz aufschließenden Wand. Teil eines Eiskeils mit einer Eisader, die aus dem Bild rechts nach oben herauszieht. Die Keilspitze unten links neben dem $2 \mathrm{~m}$ langen Maßstab endet 2,7 m unter der Oberfläche. Links oben ein glaziales Geschiebe als letzter Rest einer Grundmoräne. Foto Sommer 1963:

Fig. 2: Former marl-pit east of the village of Barmke (nr. 12, fig. 1). Part of an ice-wedge cast net with an ice-wedge and a former ice-filled vain, similar to that former ice-vain visible in fig. 3 .

Strukturen, Solifluktionsschuttdecken und Kryoturbationsformen im Vordergrund des Interesses standen. Obwohl bereits in mehreren anderen Beiträgen (Brosche \& WaLther 1978; 1991: 29 f.) einige Bemerkungen zu Eiskeilen und Eiskeilgenerationen im Untersuchungsgebiet gemacht worden sind, erscheint es reizvoll und sinnvoll, die seit gut 30 Jahren in Ostfalen gesammelten Beobachtungen vor allem zum Formenschatz der Eiskeile zu dokumentieren, zu analysieren und zu datieren. Dies um so mehr, als der größte Teil der Aufschlüsse mittlerweile in der früheren Form nicht mehr existiert. Auf die Abbildung und Beschreibung einer großen Anzahl von „normalen" Eiskeilen wird hier weitgehend verzichtet; dafür wird auf einige Sonderformen von eiskeilähnlichen Gebilden, besonders tief reichende Eiskeile und mehrphasig entstandene Eiskeilpseu- domorphosen eingegangen werden, die nicht nur von regionaler Bedeutung sind, sondern in dieser Form kaum oder noch nie dokumentiert worden sind.

Aus dem nördlichen Harzvorland liegen etliche eindrucksvolle Abbildungen zu Eiskeilpseudomorphosen von Ludwig (1958) und BRÜNING (1956 - 1959) vor, allerdings aus dem Bereich der ehemaligen DDR. In den Karten von Poser (1947 - 1951), neuerdings von WASHBURN 1979, 284 - 292, zusammenfassend dargestellt, und in der Karte von KARTE \& LIEDTKE (1979 - veröffentlicht in WASHBURN 1979, S. 300) werden noch keine Eiskeile vermerkt, auf der Karte von KaISER (1979) - veröffentlicht in WASHBURN (1979: 29) - nur die von Mania (1965), LudwIG (1958) und BRÜNING $(1956,1959)$ veröffentlichten Formen. Auf einen ausführlichen Literaturüberblick wird hier verzichtet (vgl. Teil I). Alle im Untersuchungsgebiet angetroffenen Eiskeilpseudomorphosen, Kryoturbationsformen, glazigenen Strukturen sowie die im Text behandelten sonstigen Periglazialerscheinungen sind in der Abb. 1 verzeichnet. Wie im Teil I (Eiszeitalter und Gegenwart Bd. 46, 1996) werden die an der Oberfläche (z. T. unter weichselzeitlichem Löß) anstehenden Grundmoränenablagerungen und glazifluviatilen Sedimente bis zum Vorliegen genauerer Untersuchungsergebnisse als saalezeitliche Sedimente der Drenthezeit angesehen. Dabei bin ich mir bewußt, daß genaue sedimentpetrographische Untersuchungen, wie sie in jüngster Zeit in einigen $\mathrm{Di}$ plomarbeiten aus dem Geologischen Institut der TH Hannover vorgelegt worden sind, eventuell zu Umdatierungen führen können. Die Begriffe Eiskeile und Eiskeilpseudomorphose werden hier synonym verwendet.

\section{Eiskeilnetze}

An zwei Lokalitäten: in der ehemaligen Gemeindemergelgrube von Barmke am Krähenberg östl. Barmke (bei Helmstedt - Nr. 12 in Abb. 1) und in der ehemaligen Gemeindekiesgrube Ahlum (östl. Wolfenbüttel - Nr. 18 in Abb. 1) wurden Eiskeilnetze gefunden. Bereits Harbort (1914) schreibt zu der Mergelgrube nordöstl. Barmke wörtlich: „Unter einer dünnen, bis $1 / 2 \mathrm{~m}$ mächtigen Decke von nordischen Sanden und Resten von Geschiebelehm zeigt sich die Oberfläche des Keupers bis zu etwa $1 \mathrm{~m}$ Tiefe durch die Einwirkung des Inlandeises vielfach gestaucht und gestört. Spalten, die oft bis $2 \mathrm{~m}$ tief hinabgehen, sind durch diluviale Sandmassen ausgefüllt."

Hier wurde im Jahre 1963 die gesamte völlig überwachsene Ostwand der Mergelgrube, die teilweise heute nur noch mit ca. $45^{\circ}$ abfällt, ausgegraben, wobei im Abstand von 6 bis 10 Metern acht Eiskeile und 


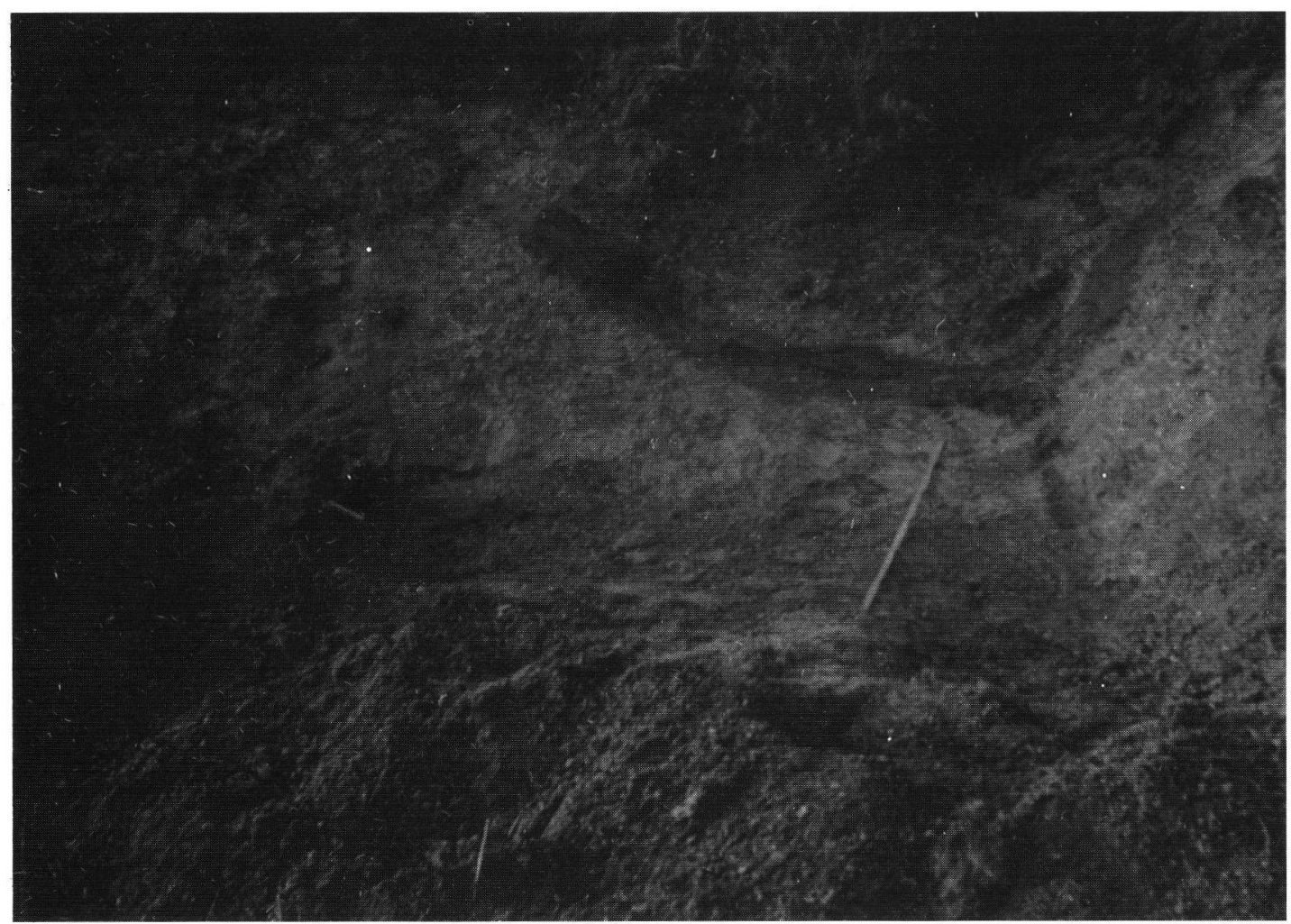

Abb. 3: Dieselbe Lokalität wie bei Abb. 2. Über dem $1 \mathrm{~m}$ langen Maßstab eine ehemalige Eisader, die heute wie alle Eiskeilpseudomorphosen bei Barmke mit rotbrauner, lehmig-toniger Grundmoräne ausgefüllt ist. Der obere Teil der Eisader endet ca. 1,0 bis 1,3 m unter der Oberfläche. Erläuterung im Text. Foto Sommer 1963.

Fig. 3: Same locality as in Fig 1. A former ice-filled vain, now filled with morainic material from the Drenthe or Elsterperiod - as all the other ice-wedge casts near Barmke - above the $1 \mathrm{~m}$ scale. Photo summer 1963.

eiskeilähnliche Strukturen zum Vorschein kamen. Man befindet sich hier bereits nördlich der nördlichen Lößgrenze in einem recht unfruchtbaren Gebiet des mittleren Keupers, in dem Pelosole lediglich Wiesen und Wald tragen. Daß hier ehemals eine elster- oder drenthezeitliche Grundmoräne verbreitet war, erkennt man an gelegentlichen spärlichen Resten einer Grundmoräne im Top des Aufschlusses, vor allem aber an den Füllmassen der Eiskeile und eiskeilähnlichen Gebilden. Sie bestehen aus einer stark tonigen, tief rotbraun gefärbten Grundmoräne, die einige nordische Geschiebe enthält. Die obersten 0,8 bis 1,0 m der gesamten Aufschlußwand sind nicht durch die Einwirkung des Inlandeises vielfach gestaucht (HARBORT 1914), sondern intensiv frostdynamisch verknetet und durch holozäne Verwitterungsprozesse „aufgeweicht" worden. Auf diese Weise wurde der ehemalige Kontakt zwischen den Eiskeilen und eiskeilähnlichen Gebilden (Abb. 2-5) und dem Liefergebiet für das Füllmaterial der Eiskeile unterbrochen oder zu einem dünnen "Schlauch“ (Abb. 4) umgestaltet.

In Abb. 2 ist der obere Teil der eiskeilähnlichen Struktur völlig vom keilfüllenden Deckmaterial ab- geschnürt worden. Die besonders dunkle Farbe des eiskeilähnlichen Gebildes resultiert aus dem Umstand, daß das Füllmaterial künstlich entfernt wurde, um nordische Geschiebe aufzuspüren. Das Gebilde in Abb. 2 besitzt einerseits einen dünnen frostspaltenartigen Fortsatz im unteren Teil, andererseits zieht ein ehemaliges „Eisblatt“ bzw. ein ehemaliger "Eisgang" nach rechts oben, wie er in Abb. 3, hier in einem weiteren Beispiel, vollständig abgebildet ist. Ähnliche Formen wurden im deutschsprachigen Raum m.W. bisher noch nicht abgebildet. Die bei KaISER (1958, Abb. 8) dokumentierten Eisgänge besitzen insofern eine gewisse Ähnlichkeit mit den hier publizierten Formen, als sie als isolierte ehemalige Eisblätter über mehrere Meter parallel zur Oberfläche verlaufen.

Die einzigen Formen aus Barmke, die herkömmlichen Eiskeilen ähneln, sind in Abb. 4 und 5 zu erkennen. Die Wand ist in beiden Fällen 60 bzw. $40^{\circ}$ geneigt. Beim Eiskeil der Abb. 4 erkennt man einerseits eine Aufspaltung der Keilspitze am unteren Bildrand, andererseits rechts neben dem Oberende des Maßstabs Reste einer lehmgefüllten ehemaligen Eisader. Der Eiskeil von Abb. 5 ragt deshalb aus der 


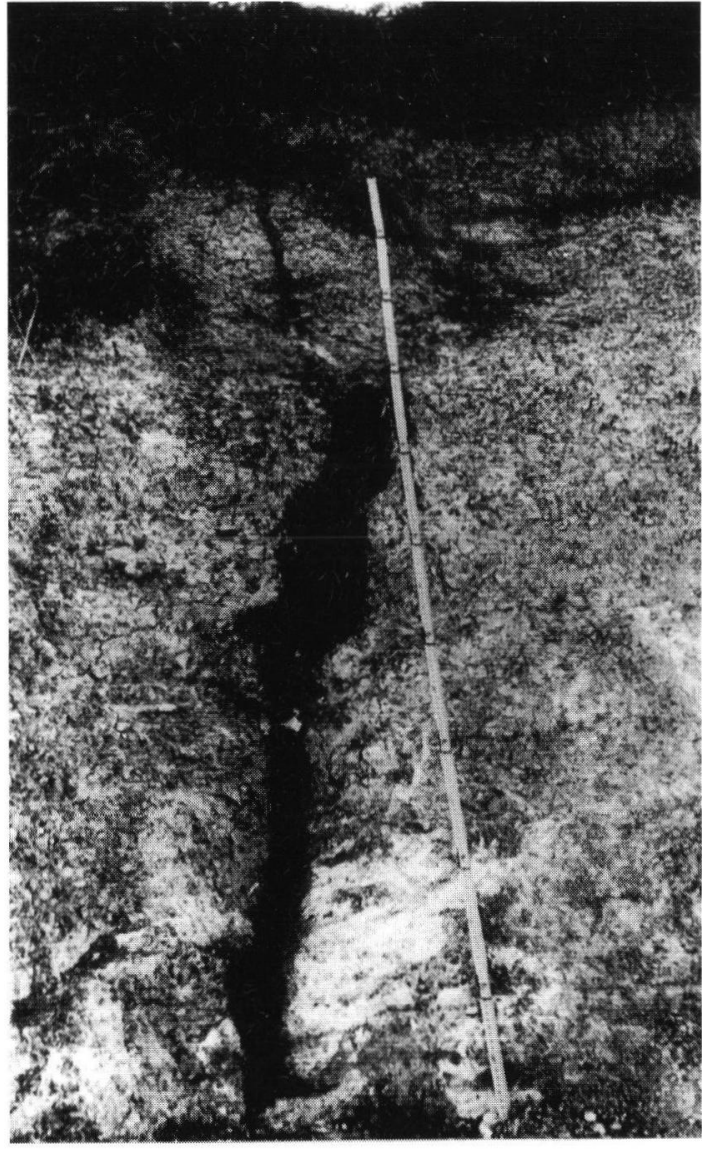

Abb. 4: Eiskeil mit zwei kleinen Spitzen von insgesamt $2 \mathrm{~m}$ Länge endet im oberen Teil an einem Eisblatt bzw. an einer Eisader (Lokalität wie bei Abb. 2 und 3) Die obersten 1,0 bis 1,3 $\mathrm{m}$ des Keupermergels sind intensiv kryoturbat verknetet, wodurch alle Eiskeiloberteile fast völlig vom Eiskeilfüllmaterial (einer wahrscheinlich drenthezeitlichen oder elsterzeitlichen Moräne) abgeschnürt wurden. Foto Sommer 1963

Fig. 4: Same locality as fig. 2 and 3. Ice-wedge cast with two little splits at its lower end. Length of the ice wedge feature $2 \mathrm{~m}$. The ice-wedge cast ends in the upper part at a former ice-vain. The upper 1,0 to $1,3 \mathrm{~m}$ of the Keuper-marl are intensively cryoturbated with the result, that all upper parts of the ice-wedge structures are separated from the Saalian or Elsterian morainic material you now find in the former ice-wedges. Photo summer 1963.

Wand heraus, weil das mürbe graue Mergelmaterial des mittleren Keupers leicht abgekratzt werden konnte, so daß die rotbraune lehmige Keilfüllung wenigstens um $10 \mathrm{~cm}$ aus der Wand herausragt. Im internen Sprachgebrauch wurde dieser Eiskeil wegen seiner Ähnlichkeit mit dem Umriß Italiens „Eiskeil Italien" genannt. In dieser einzigartigen Form präsentiert sich der Lehmkeil allerdings nur, weil die Wand hier nur ca. $40^{\circ}$ geneigt ist.

Aufgrund der Abschnürung des Keilfüllmaterials durch den Vorgang von Kryoturbation bzw. des viel- tausendfachen Gefrier- und Tauprozesses in den oberen 0,8 - 1,0 Metern erscheint es sinnvoll, die Eiskeilbildung und spätere -verfüllung zumindest in die vorletzte Kaltzeit zu stellen. Mit dieser Deutung verträgt sich auch die Tatsache, daß die vermutlich drenthezeitliche oder elsterzeitliche Grundmoräne trotz flacher Oberflächenformen weitgehend abgetragen worden ist. Die Eiskeilfüllung muß zu einer Zeit erfolgt sein, als noch genügend Grundmoräne vorhanden war. Wären die Eiskeile erst am Ende der Weichselzeit oder in der Weichseleiszeit fossil geworden, so hätte wahrscheinlich nicht genügend Eiskeilfüllmaterial zur Verfügung gestanden. Eine Mehrphasigkeit der Bildung der Eiskeile und eiskeilähnlichen Gebilde bei Barmke ließ sich nicht feststellen (s. u.).

Das zweite Eiskeilspaltennetz innerhalb des Untersuchungsgebietes war in den 60er Jahren am östlichen Ortsrand von Ahlum (östl. Wolfenbüttel) in der ehemaligen Gemeindekiesgrube aufgeschlossen (Nr. 18 in Abb. 1). Ein langer W-E streichender Kiesrücken, der durch zur Oker entwässernde Bäche herausgeformt wurde, wird hier von einer sehr dünnen, kaum 1 m Mächtigkeit messenden weichselzeitlichen Lößdecke überzogen. Die ausgebeuteten Kiese weisen eine Mächtigkeit von mindestens 7-8 Metern auf. Im Gegensatz zum Eiskeilspaltennetz von Barmke erreichen die hier aufgeschlossenen Eiskeile durchweg die recht selten vorkommende Länge von 6 Metern (Abb. 6). Der Abstand der einzelnen, überwiegend mit glazifluvialen Kiesen gefüllten Eiskeile beträgt zwischen 8 und 12 Metern (Abb. 7). Wie Abb. 5 zeigt, fungieren als Füllmaterial des nach unten sich verschmälernden Eiskeils überwiegend die glazifluvialen Kiese, die auch die Hauptmasse der Aufschlußbwand darstellen. Lediglich der oberste Teil des Füllmaterials bestand aus lehmigen moränalen Sedimenten, wahrscheinlich aus der Drenthezeit. Von einer Mehrphasigkeit der Eiskeilbildung kann im Gegensatz zu anderen Lokalitäten innerhalb des Untersuchungsgebietes (s. u.) nicht sicher gesprochen werden. Da kein weichselzeitlicher LÖß als Füllmaterial gefunden wurde, wird der Eiskeil als drenthezeitlich oder älter eingestuft.

\section{Vorkommen einphasiger, mäßig tiefer Eiskeile}

Die überwiegende Anzahl der aufgefundenen Eiskeile gehört in die Gruppe der einphasig entstandenen, 1 bis 2,5 m tiefen Eiskeilpseudomorphosen. In dieser Gruppe lassen sich mit einiger Sicherheit jüngere (weichseleiszeitliche) und ältere (wahrscheinlich saalezeitliche) Formen unterscheiden. Innerhalb des weichselzeitlichen Lößprofils im Tagebau Alversdorf sind sogar mehrere Eiskeilstockwerke anzutreffen (Brosche \& Walther 1991: 28 f., Foto 3 und 4). 


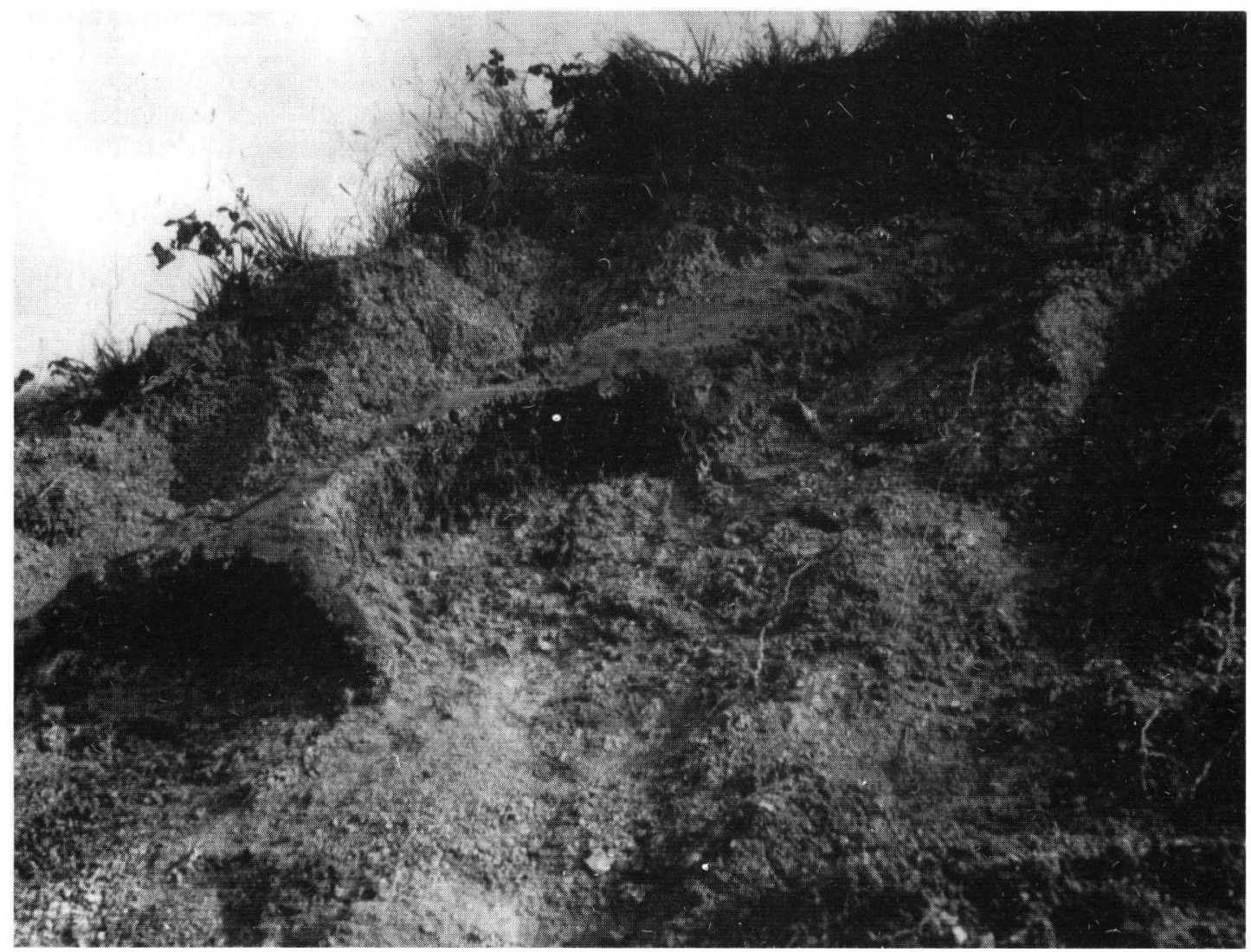

Abb. 5: Der beeindruckendste Eiskeil, der wegen seiner Form „Eiskeil Italien“ genannt wird. Die Wand ist hier nur ca. $40^{\circ}$ geneigt; deshalb konnte die moränale Füllmasse in dieser Weise ausgegraben werden. Dieser Eiskeil besitzt ebenfalls zwei Spitzen. Tiefe des Keiles 1,5 m. Foto Sommer 1963.

Fig. 5: Same locality as in fig $1-3$. The best developed ice-wedge cast, which got the name ice-wedge „Italia“ from ist similarity to Italy's form. Inclination of the pit wall ca. $40^{\circ}$. Therefore the morainic filling could be excavated; this ice-wedge cast also posesses two splits at ist lower end. Depth of the structure $1.5 \mathrm{~m}$. Photo summer 1963.

Die Kiesgrube Evers am Nordschacht $5 \mathrm{~km}$ westl. Helmstedt südl. der Bundesstraße 1, Nr. 10 in Abb. 1) ist seit 30 Jahren eine Fundgrube, was das Auftreten periglazialer Phänomene betrifft. Glazifluviale Sande und Kiese in einer Mächtigkeit von 10-15 m über eozänen Sanden bilden die Hauptmasse des Aufschlusses. Überdeckt werden die glazifluvialen Sande und Kiese von einer teils sandig-kiesigen Grundmoräne (Abb. 8), teils von einer lehmig-tonigsandig-kiesigen Grundmoräne, die ihrerseits von unterschiedlich mächtigem, ungegliedertem weichselzeitlichem Löß überzogen wird. Eine z.T. deutlich entwickelte Parabraunerde ist auf dem Löß ausgebildet (auf Abb. 8 abgeräumt). Der gesamte quartäre Sedimentkörper, der vermutlich saalezeitlichen (drenthezeitlichen) Alters ist, weist an seiner Basis Unebenheiten, ja deutliche Sprünge mit 0,5 bis $1 \mathrm{~m}$ Vertikalversetzungen auf, was auf quartäre Tektonik hinweist. Innerhalb des quartären Sedimentkörpers gibt es hierfür allerdings keine Anzeichen.

Der in Abb. 8 abgebildete Eiskeil enthält als Füllmaterial eine rotbraun gefärbte, steinig-kiesige Grund- moräne mit schwach lehmigen Anteilen, vielleicht im unteren Teil auch glazifluviale Sedimente. Die Grundmoräne entstammt offenbar der Schicht 1. Die rötlich-braune Verfärbung des Füllmaterials findet sich im unteren Teil der Grundmoräne rechts und links der Eiskeilschultern ebenfalls wieder und ist in Ostfalen typisch für Grundmoränen im Altmoränengebiet. Folgende Deutung des Aufschlußbildes scheint mir plausibel zu sein: In den glazifluvialen Sanden und Kiesen (Schicht 2) hatte sich vor Herannahen des Eises ein Eiskeilnetz entwickelt, welches von dem vorstoßenden Eis überdeckt wurde. Wahrscheinlich handelte es sich um drenthezeitliche Vorgänge. Nach dem Abtauen des Eises konnte die im Rahmen des Eisabtauvorganges abgelagerte Grundmoräne als Füllmaterial der Eiskeilpseudomorphose fungieren. Es handelt sich also in jedem Falle um einen präweichselzeitlichen Eiskeil, dessen Füllmate-

1) Im unteren Teil dieses ca. $1 \mathrm{~m}$ langen Lößkeiles wurde die links und rechts vom Keil befindliche Löß-Schmutztapete ebenso wenig entfernt wie ca. $2 \mathrm{~m}$ unterhalb des Tops des Aufschlusses über der Kies-Eiskeilfüllung. 


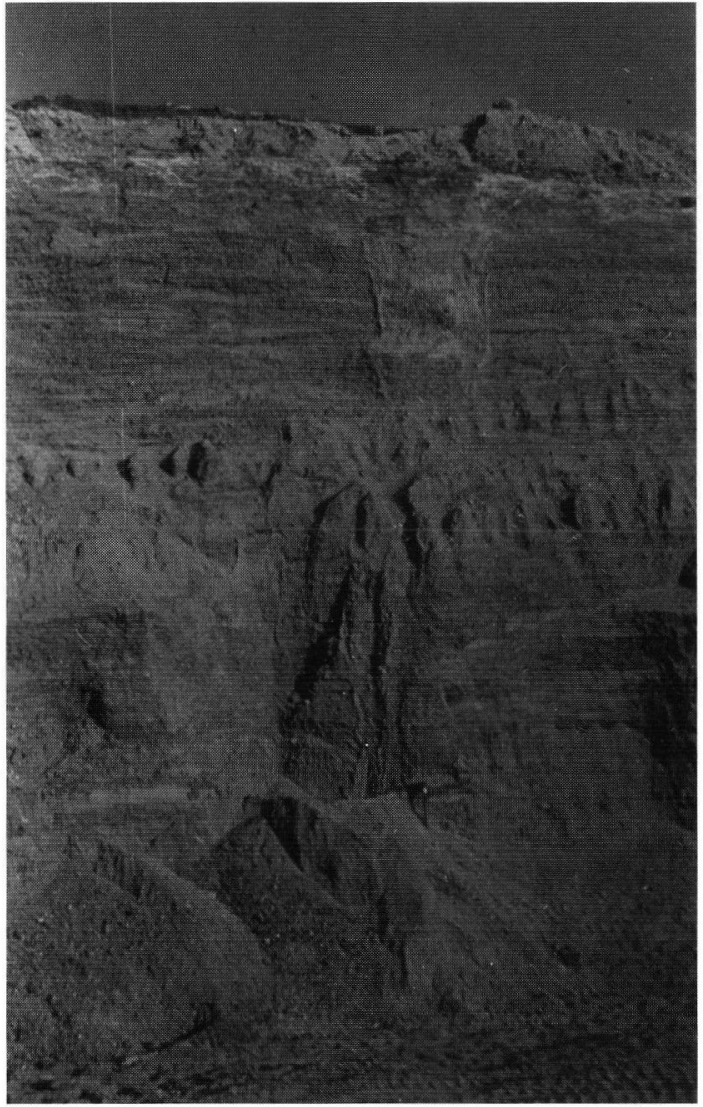

Abb. 6: Circa $6 \mathrm{~m}$ langer Eiskeil als Teil eines Eiskeilspaltennetzes in der ehemaligen Gemeindekiesgrube Ahlum am östlichen Dorfrand (Nr. 18 in Karte 1). Maßstab ein Klappspaten im unteren Teil rechts neben dem Keilende. Breite an der Keilschulter 0,6 bis 0,7 m. Füllmasse sind die den Aufschluß beherrschenden glazifluvialen Sande, Kiese und Schotter, im oberen Teil auch Reste einer rotbraun verwitterten, wahrscheinlich drenthezeitlichen Grundmoräne. Eine ehemalige dünne Lößschicht wurde künstlich entfernt. Foto Sommer 1963:

Fig. 6: Ice-wedge cast with a length of $6 \mathrm{~m}$ as part of a greater net of ice-wedge casts (nr. 18, map 1). Locality of figure 6 is the former village gravel pit of Ahlum, east of Wolfenbüttel. Asscale a little spade with a length of $0.5 \mathrm{~m}$ is used, visible at the right hand side of the lower end of the ice-wedge structure. The upper part of the structure is $0,6-0,7 \mathrm{~m}$ large. The ice-wedge is mainly filled with the same glacifluvial material of the Drenthe or Elster time, which forms the wall of the whole pit. In the upper part of the structure there can be seen a rest of a redbrown morainic sediment. Weichselian loess is moved away above the structure. Photo summer 1966.

rial besonders in der Eemzeit kräftig verwittern konnte. Von diesem Eiskeiltyp gab es 1965 weitere Beispiele in derselben Grube, die sich im Februar 1994 durch weitere Funde bestätigten (auf Abbildungen wird hier verzichtet). Auf einen mehrphasigen Eiskeil an der gleichen Wand wird im nächsten Kapitel eingegangen.

An der Nordwand des gleichen Aufschlusses fanden sich im Februar 1994 mit anderem Füllmaterial versehene jüngere Formen, allerdings an der in knapp 30 Jahren ein wenig nach Norden zurückverlagerten Wand. Abb. 9 zeigt ein Beispiel des eher plumpen Eiskeiltyps, der mit ungegliedertem weichselzeitlichem Löß gefüllt ist und nur 1-1,3 m tief in die wahrscheinlich drenthezeitliche Grundmoräne hineingreift. Die Breite am oberen Ende entspricht in etwa der maximalen Tiefe. Es besteht kein Zweifel daran, daß es sich hierbei um ein weichselzeitliches Gebilde handelt. Die Form ist nicht ungewöhnlich, denn in Winnigstedt (südl. Schöppenstedt nahe der ehemaligen Zonengrenze) und in der ehemaligen Ziegeleigrube Roklum fanden sich hier nicht abgebildete ähnliche Eiskeile.

$\mathrm{Zu}$ dieser Gruppe der einphasigen mäßig langen Eiskeile gehören mehrere Vorkommen in der ehemaligen Ziegeleigrube Roklum (Nr. 17, Abb. 1), an der Nordwand des ehemaligen Tagebaus Treue, an der Südwand des Tagebaus Helmstedt (Nr. 7, Karte 1, Nr. 8, Abb. 1), im Tagebau Alversdorf nahe dem Stellwerk (Nr. 9, Abb. 1) (Brosche \& Walther 1991: 29 f.), in Baugruben südl. des Ludgerikreuzes bei Helmstedt (Nr. 1, Abb. 1) und in der Gemeindekiesgrube bei Winnigstedt (Nr. 16, Abb. 1). Nur drei Formen seien hier besprochen und abgebildet. Zunächst erscheint mir ein merkwürdiges, mit Löß gefülltes Gebilde an der E-Wand der ehemaligen Ziegeleigrube Roklum bemerkenswert zu sein (Abb. 10). Es ist wohl eine Definitionsfrage, ob man hier von zwei schräg abwärts ziehenden Eisadern oder von zwei schmalen Eiskeilen mit einer gemeinsamen Wurzel spricht. Die Gebilde sind 1,5 bis $2 \mathrm{~m}$ lang, mit hellem, kalkhaltigem, jungweichselzeitlichem Löß gefüllt und reichen in kreidezeitlichen grüngrauen Neokomton hinein. Mit ziemlicher Sicherheit kann aufgrund vergleichender lößstratigraphischer Untersuchungen von einem Alter jünger als das Deenekamp-Interstadial ausgegangen werden; denn nur im jüngeren Jungweichsellöß treten in dieser Gegend die hellgelben kalkhaltigen Lösse auf, die hier das Füllmaterial stellen.

Wegen seiner klassischen Form sei ein Eiskeil von der Nordwand des ehemaligen Tagebaus Treue abgebildet und kurz besprochen (Abb. 10 - Nr. 7, Abb. 1). Es handelt sich um eine lößgefüllte Eiskeil-pseudomorphose von ca. $2 \mathrm{~m}$ Länge, die in mittel-oligozänen Septarienton mit Septarien (vgl. rechts unten neben dem Maßstab, einem Klappspaten) eingreift. Links vom oberen Ende des Eiskeils befindet sich eine Krotowine, rechts vom oberen Ende des Eiskeils und über dem Eiskeil beobachtet man z.T. Löß, der wahrscheinlich durch Tiefpflügen erfaßt worden ist. Beide Keilschultern sind mäßig aufgebogen, wie aus schwachen Farbunterschieden des angrenzenden Materials erschlossen werden kann. Bezüglich der zeitlichen Einstufung gelten die gleichen Aussagen, 


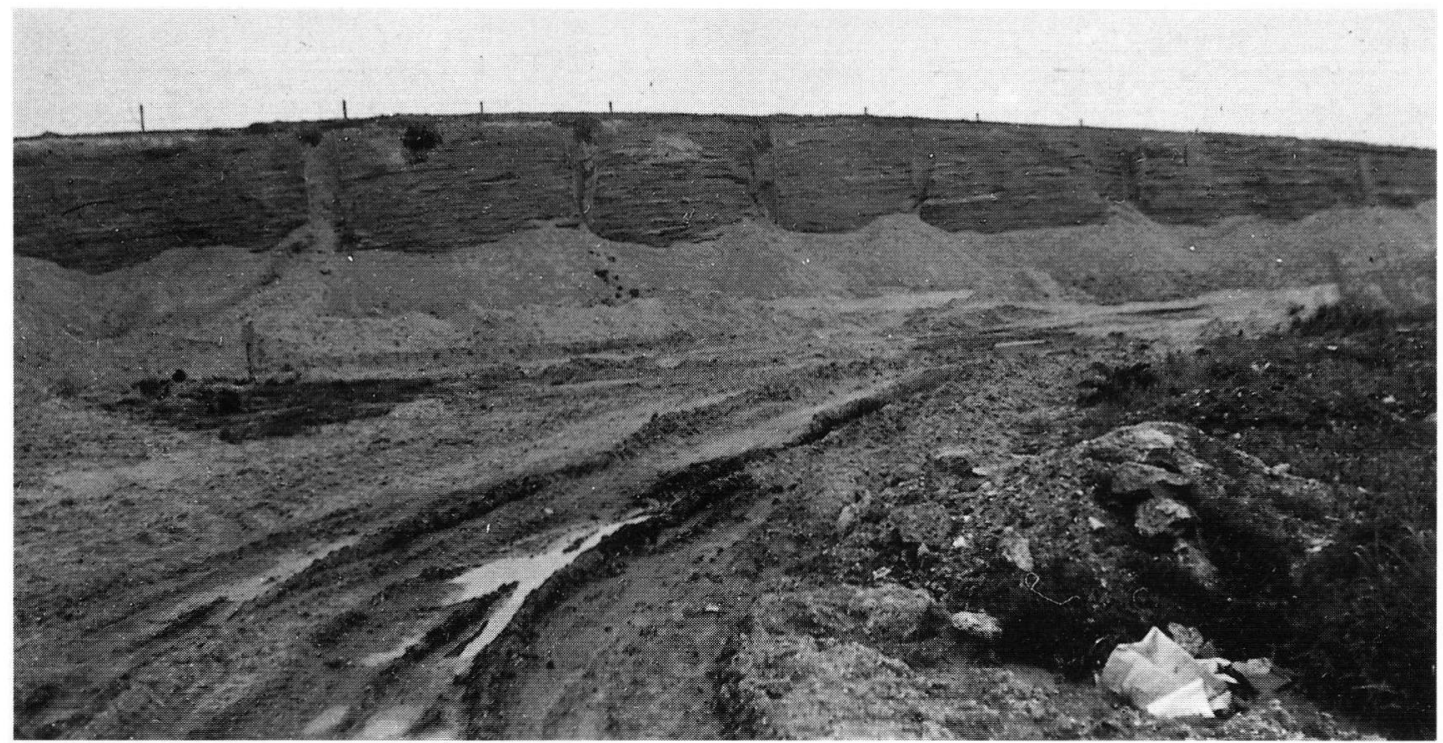

Abb. 7: Das bedeutendste Eiskeilspaltennetz des Untersuchungsgebietes an der Ostwand der ehemaligen Gemeindekiesgrube Ahlum. Wie in Winnigstedt am Nordrand des Magdeburg-Oscherslebener Urstromtales und bei Wegeleben am Südrand des gleichen Urstromtales auch hier ein mächtiger W - E streichender Kiesrücken aus glazifluvialem Material. Mindestens 6 Kieskeile von je 6 m Länge erkennbar. Foto Sommer 1963.

Fig. 7: The most important net of ice-wedge casts in the observation area of eastern Lower Saxony. Same locality as fig 5 (nr. 18, map 1). Eastern wall of the gravel pit of Ahlum, east of Wolfenbüttel. At least 6 big ice-wedge casts with a higth of $6 \mathrm{~m}$ are visible in glacifluvial sediments extending in W-E-direction and building up a broad gravel wall as at both sides of the Magdeburg-OscherslebenerUrstromtal nearby. Photo summer 1963.

die zum zuletzt behandelten Eiskeil gemacht worden sind.

Der in Abb. 12 dargestellte Eiskeil vom Südrand des Tagebaus Helmstedt gehört, was seine zeitliche Einstufung als hochjungweichselzeitliches Gebilde anbetrifft, in die gleiche Gruppe wie die beiden zuvor behandelten Formen (Nr. 8, Abb. 1). Auch hier fungiert deutlich heller gelber Jungweichsellöß als Füllmaterial. Dieser helle gelbe kalkhaltige Löß wurde als ungeschichteter Fremdlöß angeweht, als oder nachdem der geschichtete Löß der Hauptmasse (Schicht 3), der viele lokale Komponenten enthält, angeweht und von einer Eiskeilbildung durchschlagen worden war. Bemerkenswert ist die Aufschleppung des geschichteten jungweichselzeitlichen Lösses an der rechten Keilschulter und die fast taschenbodenartige Verformung des gelben, kalkhaltigen jungweichselzeitichen Lösses (Schicht 2) rechts neben dem oberen Teil des Eiskeils. Die schwache Bodenbildung im Lößpaket 1 greift über den Löß der Schicht 2 hinweg. Bei diesem Lößkeil handelt es sich, was bisher noch kaum beobachtet worden ist, um eine Kombination aus epigenetischem Eiskeil und syngenetischer schmaler, unterschiedlich gut erkennbarer Frostspalte (vgl. auch Gallwitz 1949; KaISER 1960: 126). Diese scheint vom unteren Rand der Abbildung aus allmählich nach oben gewachsen zu sein. Zum Alter dieser syngenetischen Frostspalte können keine sicheren Aussagen gemacht werden, da das Alter des Lösses von Schicht 3 und des unten in Höhe des Maßstabs sichtbaren Lösses nicht sicher eingestuft werden kann.

\section{Mehrphasig entstandene Eiskeilpseudomorphosen}

Der in Abb. 13 dargestellte $5 \mathrm{~m}$ lange Eiskeil wurde 1966 in der schon mehrfach erwähnten Kiesgrube Evers am Nordschacht $5 \mathrm{~km}$ westl. von Helmstedt angetroffen (Nr. 10 in Abb. 1). Nirgendwo sonst waren die staffelbruchartigen Versetzungen der dem Eiskeil benachbarten Schichten so deutlich ausgeprägt wie in diesem Beispiel. Am Top des Aufschlusses sind als dunkler Streifen letzte Reste einer rotbraun verwitterten Grundmoräne (wahrscheinlich aus der Drenthezeit) erkennbar, während der gesamte weichselzeitliche Löß hier künstlich abgeräumt worden ist. Die Hauptmasse der Aufschlußwand stellen glazifluviale Sande und Kiese mit Kreuzschichtung oder bogiger Schrägschichtung. Während die Hauptmasse der Eiskeilfüllung in einen älteren Teil der Saalekaltzeit zu stellen sind, läßt sich im oberen Teil des Eiskeils eine zweite dunklere Füllmasse erkennen, die überwiegend aus Moränenmaterial besteht. Dies weist auf eine Reaktivierung der Eiskeilbildung in einem jüngeren Stadium des Drenthe-Stadiums hin, zu einem Zeitpunkt, bevor das Eis seine Grundmoräne ablagerte. 


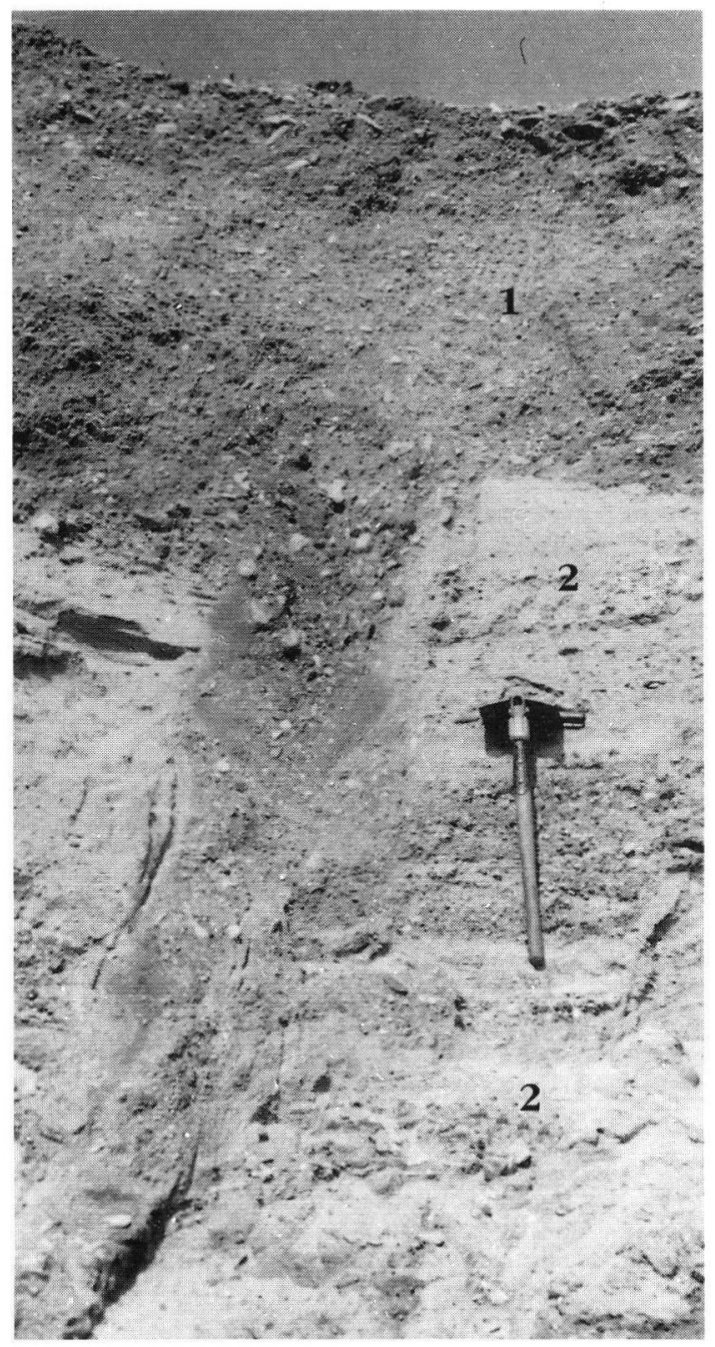

Abb. 8: Über $2 \mathrm{~m}$ langer Eiskeil an der Nordwand der Kiesgrube Evers am Nordschacht $(5 \mathrm{~km}$ westl. Helmstedt, südl der B 1 - Nr. 10 der Karte 1). Breite an der Keilöffnung 40 $45 \mathrm{~cm}$. Über Schicht 1, einer steinig-kiesigen Grundmoräne, wurde der weichselzeitliche Löß künstlich entfernt. Sowohl Schicht 1 als auch Eiskeilfüllmaterial durch Eisenmobilisierung rotbraun verwittert und verfestigt gegenüber den glazifluvialen Sanden, Kiesen und Schottern (Schicht 2). Foto Sommer 1965

Fig. 8: A $2 \mathrm{~m}$ long ice-wedge cast at the northern part of the great gravel pit of Evers near the Nordschacht, $5 \mathrm{~km}$ west of Helmstedt (nr. 10, fig. 1). Upper part of the gravel-filled structure is $0,4-0,45 \mathrm{~m}$ large. In the top of the pit Weichselian loess and part of a gravel morainic sediment were removed. All sediments filling the ice-wedge cast are cementated by mobilized iron. Main sediment of the pit (nr. 2, fig. 1) consists of glacifluvial sediments probably of Drenthe-age. Photo summer 1965.

Wäre diese Deutung richtig, so wären auch für den älteren Abschnitt der Saalekaltzeit (wahrscheinlich der Drenthezeit), was nicht verwunderlich ist, mehrere Phasen des Dauerfrostbodenaufbaus und Dauerfrostbodenabbaus erwiesen. In etwas modifizier-

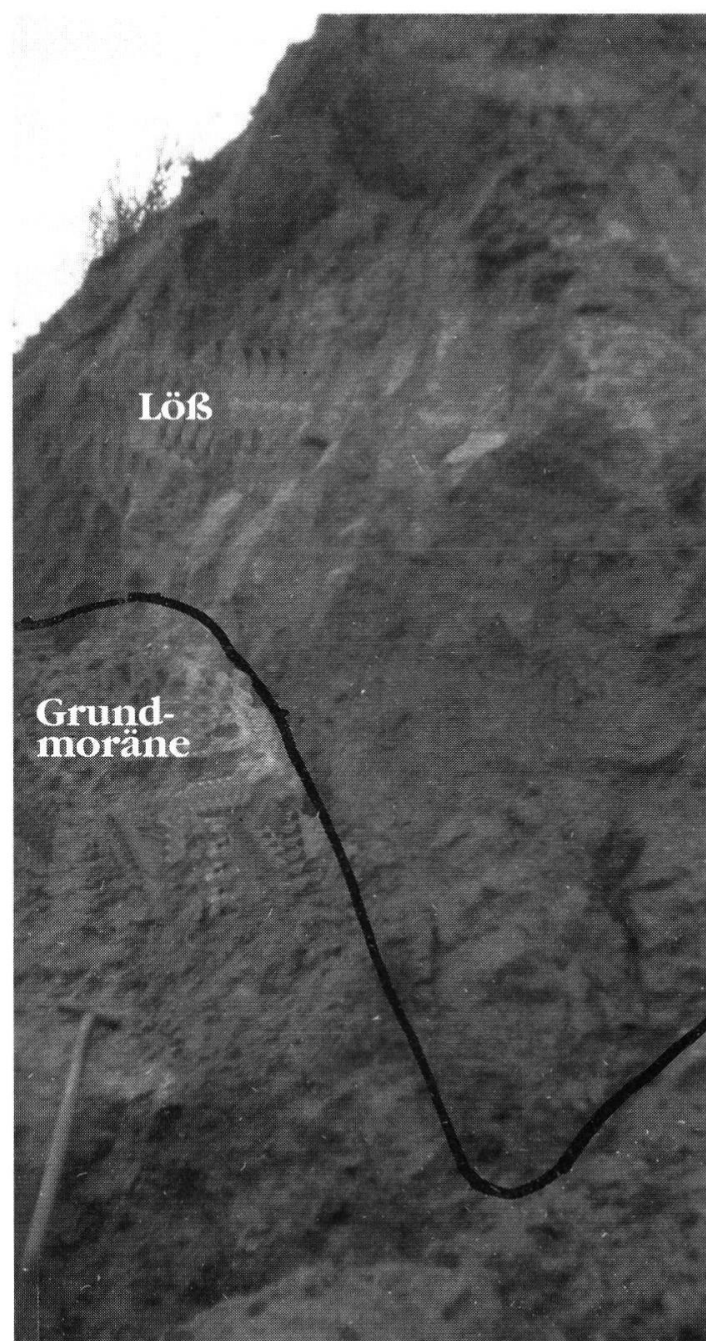

Abb. 9: Massiger, recht breiter und nicht sehr tiefer Eiskeil mit Lößfüllung an der Nordwand der Kiesgrube Evers am Nordschacht $5 \mathrm{~km}$ westl. von Helmstedt. Maßstab ein $1 \mathrm{~m}$ langer Spaten. Foto Februar 1994.

Fig. 9: In the upper part very large and at the whole not very deep ice-wedge cast, which was often found as a special example of a Weichselian ice-wedge, mostly filled with Weichselian loess (nr.10, fig. 1). Northern wall ot the huge gravel pit at the Nordschacht near Helmstedt. As scale serves a $1 \mathrm{~m}$ lang spade. Photo February 1994

ter Form läßt sich das gleiche Phänomen der Reaktivierung einer bereits mit älterem Material gefüllten Eiskeilspalte in der Gemeindekiesgrube Winnigstedt (südl. Schöppenstedt) beobachten (Abb. 13 - Nr. 16 in Abb. 1). Hier sind 8-10 m mächtige glazifluviatile Sande und Kiese aufgeschlossen; die steinigen Anteile bestehen überwiegend aus Muschelkalkgeröllen, die aus der Asse-Fortsetzung oder aus dem Elm stammen. Ein langer Kiesrücken verläuft hier in WE-Richtung parallel zum Magdeburg-Oscherslebener Urstromtal. Die Abbildung 13 läßt diesen in N-S 


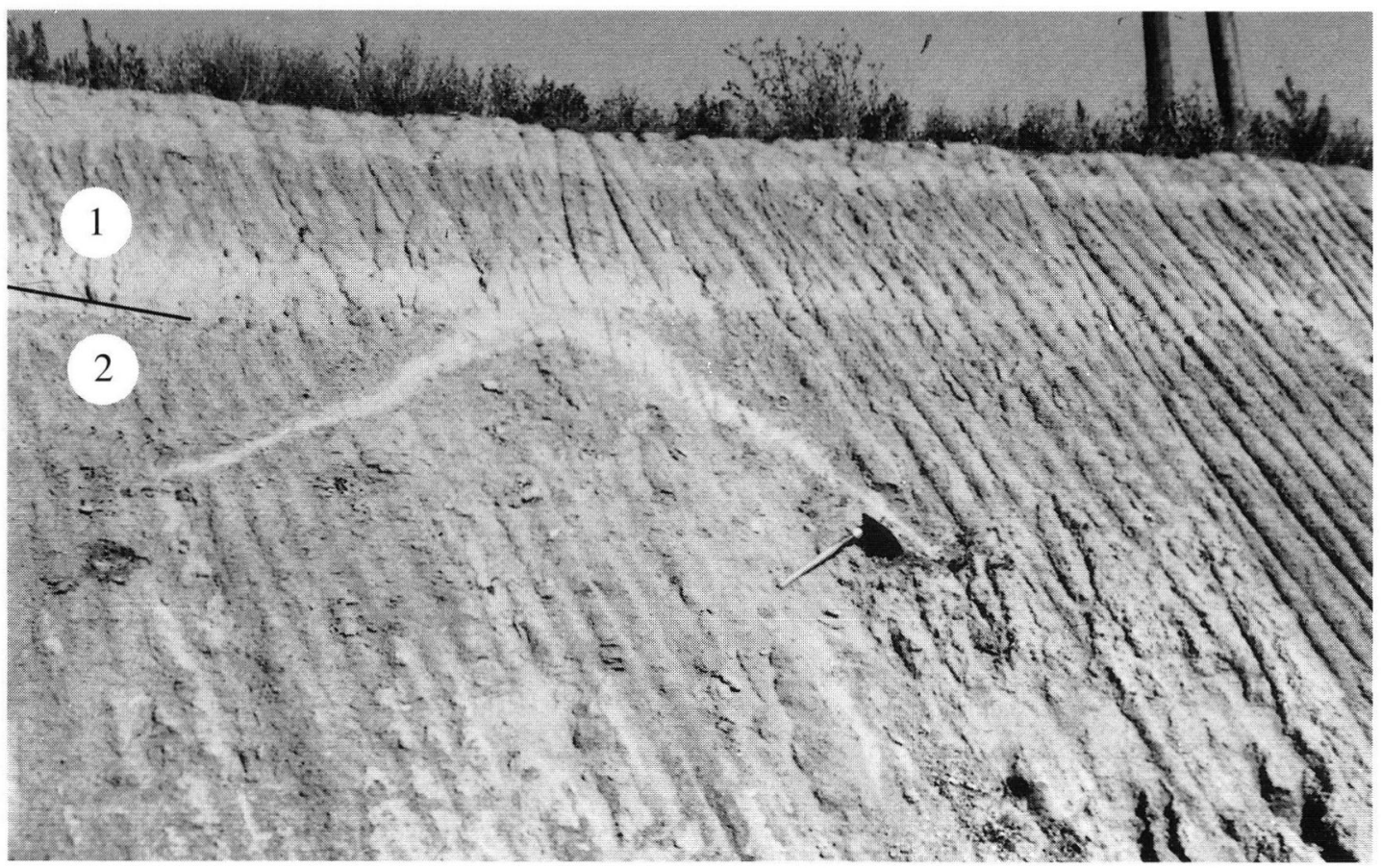

Abb. 10: Zwei mit Löß gefüllte Eisadern bzw. Eiskeile von 2 Meter und knapp 2 Meter Länge in der ehemaligen Ziegeleigrube Roklum (südl. Schöppenstedt, Nr. 17, Karte 1). Der hochweichselzeitliche gelbe Löß (1) liefert das Füllmaterial für die eiskeilähnlichen Strukturen, die in Neokomton hineinreichen. Wand etwa $45^{\circ}$ geneigt. Foto Sommer 1966.

Fig. 10: Two former ice-vains or ice-wedge casts filled with very young upper Weichselian loess with a length of about $2 \mathrm{~m}$. Former pit of the brick factory of Roklum (south of Schöppenstedt - nr. 17, fig. 2). The ice-wedge cast penetrates in neocomic clay. Inclination of the wall ca. $45^{\circ}$. Photo summer 1966.

Richtung aufgeschlossenen Kiesrücken erkennen. Die Eiskeilfüllung stellt sich besonders im unteren Teil (neben und über dem Zollstock) als Positivform in der Kieswand dar. Sie reicht jedoch noch bis 0,4 $0,5 \mathrm{~m}$ unterhalb de Zollstocks bis etwa in Höhe des rechten Fußes meines Sohnes hinab. Die Positivform der Eiskeilfüllung erklärt sich aus einer intensiveren Verfestigung des Eiskeilfüllmaterials, die offenbar durch eine stärkere Wasserabfuhr und Kalkausfällung in der Eiskeilspalte hervorgerufen wurde. Im obersten Meter besteht der Eiskeil überwiegend aus weichselzeitlichem Löß (an dunkler Farbe erkennbar); darunter folgt als Keilfüllung Kies und ein Gemisch aus Kies und Löß. Ebenso wie die Eiskeile im westlich gelegenen Ahlum (Abb. 6 und 7) handelt es sich um ein 5,0 - 5,5 m langes Eiskeilgebilde, dem sich ca. $20 \mathrm{~m}$ weiter nördlich (hier nicht abgebildet) ein zweiter, nur gut $1 \mathrm{~m}$ tief reichender Lößkeil zugesellt. Dieser ähnelt im Prinzip dem in Abb. 9 sichtbaren plumpen Keil vom Nordschacht westlich Helmstedt. Die Deutung der hier vorgefundenen Verhältnisse lautet folgendermaßen: Die Haupteiskeilbildung geschah - ein drenthezeitliches Alter der glazfluviatilen Sande, Schotter und Kiese vorausgesetzt - noch innerhalb der Saalekaltzeit. Wegen der sich deutlich nach unten verjüngenden Keilform dürfte wie in allen anderen dargestellten Beispielen eine epigenetische Eiskeilbildung vorgelegen haben. Später in der Weichselkaltzeit muß es zu einer schwachen Wiederbelebung der Keilbildung gekommen sein, wobei die entstandene Hohlform später mit weichselzeitlichem Löß aufgefüllt wurde. Bei der braunen Lehmmasse 0.6 - 0,7 m unterhalb der Oberfläche kann es sich z.T. um eine Schmutztapete handeln (vgl. Fußnote 1, S. 95).

Eine weitere wahrscheinlich mehrphasige Eiskeilstruktur in einer weichselzeitlichen Lößserie mit altweichselzeitlichem Humusmaterial als ältester und jungweichselzeitlichem Löß als jüngster Keilfüllung wurde von Brosche \& Walther (1991: 29 f. und Foto 4) im Tagebau Alversdorf gefunden und beschrieben (Nr. 9, Abb. 1).

\section{Kleinkeile und Kissenböden}

Als Kleinteile bezeichneten RoHDENBURG (1966:166) und ROHDEnburg \& Meyer (1966) keilförmige Gebilde mitten in einem vielgliedrigen Lößprofil. Das einzige Vorkommen innerhalb unseres erweiterten Untersuchungsgebietes fand ich zusammen mit Herrn Priv.-Doz. Dr. M. Walther, Berlin, in der ehemaligen Ziegeleigrube Sudhoff bei Einbeck (Abb. 15). Der 


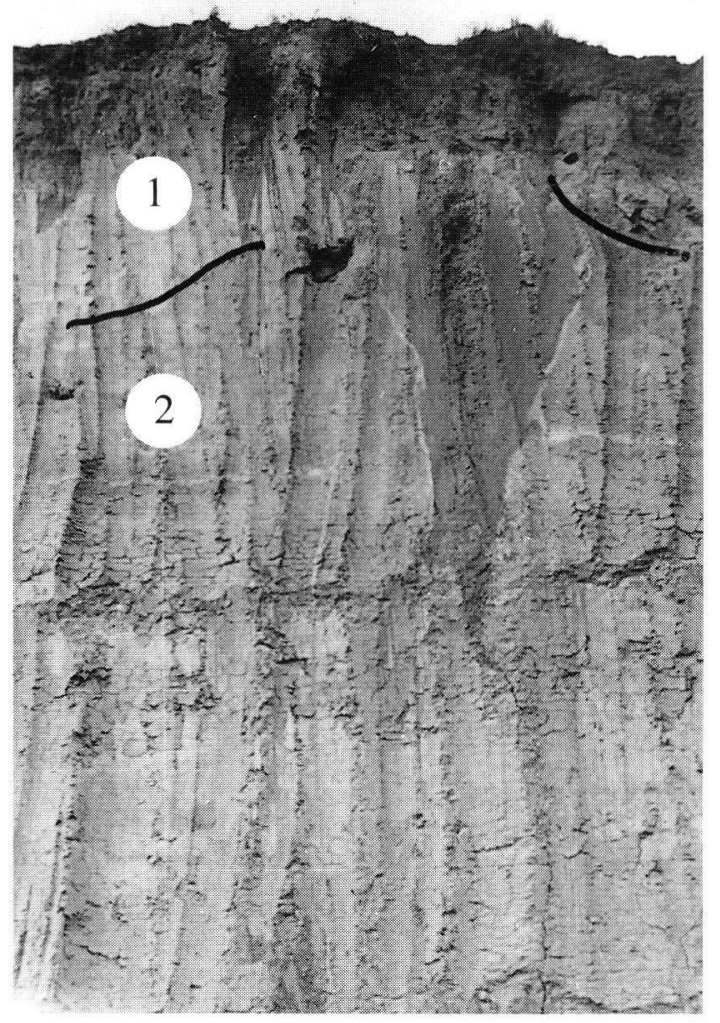

Abb. 11: Lößgefüllter Eiskeil von ca. 2 m Länge an der Nordwand des Tagesbaus Treue der BKB Helmstedt. Etwa auf der Höhe des ehemaligen Dorfes Runstedt. Wandhöhe 8-10 Meter. Füllmaterial des Eiskeils ist hochglazialer weichselzeitlicher gelber (hier feuchter) Löß, der auch in dünner Decke als Schicht 1 weitflächig verbreitet ist. An Keilschulter links ist dieser Löß hochgedrückt. Schicht 2 sind mitteloligozäne, glaukonitische Septarientone mit Krotowine nahe der Grenze von (1) zu (2). Foto Sommer 1965.

Fig. 11: Loess-filled ice-wedge cast with a length of $2 \mathrm{~m}$. Northern wall of the mining-field Treue of the Braunschweigische Kohlenbergwerke Helmstedt (nr. 7, fig. 1). Hight of the pit 8 $10 \mathrm{~m}$. As filling material acts high glacial Weichselian yellow chalky loess, which also covers the whole surface (1). At the shoulder of the left upper part of the ice-wedge cast this yellow loess is pressed upwards. Layer 2 consists of middle Oligocene "Septarienton". A Krotowine is visible in its upper part. Photo summer 1965.

untere Rand des E 4-Naßbodens des Jungweichsellösses (nach der Nomenklatur von SCHÖNHALS, RoHdenburg \& Semmel 1964) weist hier 10-20 cm lange keilartige Strukturen auf, wie sie bisher nur bei Rohdenburg (1966: 166) abgebildet wurden. Mit ROHDENBURG wird angenommen, daß es sich hierbei nicht um Dauerfrostbodenanzeiger handelt, sondern daß hier lediglich temporär innerhalb einer

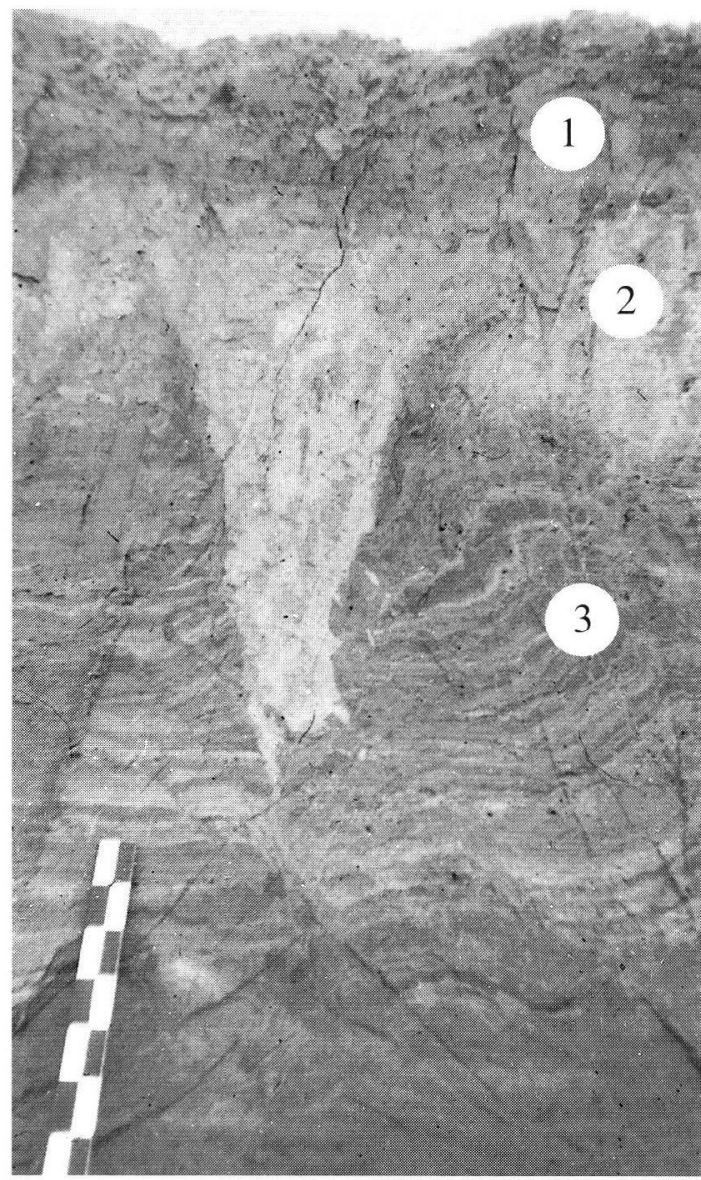

Abb. 12: Ca. 1 m langer lößgefüllter Eiskeil an der S-Wand des Tagebaus Helmstedt der BKB Helmstedt (Nr. 8, Karte 1). Gelber kalkhaltiger Löß des oberen weichselzeitlichen Hochglazials (Schicht 2) als Füllmaterial. Oberste Lößschichten über Schicht 1 künstlich entfernt. Deutliche Aufbiegungen in geschichtetem Jungweichsellöß (3). Linke Keilspitze setzt sich als Frostspalte noch fast $1 \mathrm{~m}$ weiter in die Tiefe fort. Obere Eiskeilgeneration i.S. ROHDEnBurgs (1966). Foto Sommer 1977.

Fig. 12: Ice-wedge cast with a length of about $1 \mathrm{~m}$, filled with very young upper Weichselian loess, situated at the southern wall of the coal field Tagebau Helmstedt (Braunschweigische Kohlebergwerke Helmstedt) (nr. 8, fig. 1). The upper loess layers above layer (1) are removed. The vicinous layers of the icewedge cast are pressed upwards. The left split of the feature continues as a very thin frost split (Frostspalte) for $1 \mathrm{~m}$ in the depth. Photo summer 1977.

Kaltphase der Weichseleiszeit kleine Spalten aufrissen, die kurz darauf als syngenetische Mikroformen mit jüngerem Löß gefüllt wurden.

Gleiches gilt auch für die Kissenbodenstrukturen, die in Abb. 16 - ebenfalls in der Ziegeleigrube Sudhoff bei Einbeck aufgenommen - zu sehen sind. Sie sind im Zuge der Entstehung kleiner syngenetischer Frostrisse gebildet worden, die sich deshalb so deutlich erkennen lassen, weil hier echte gelbe „Fremd- 


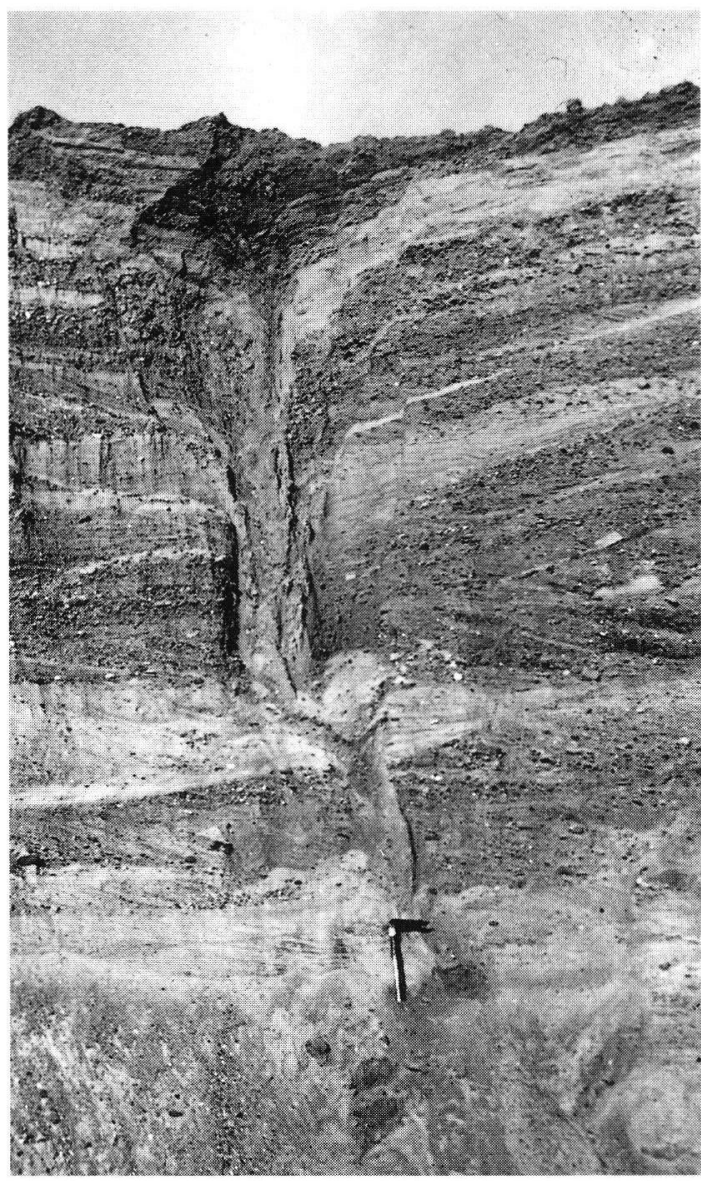

Abb. 13: Ca. 5 m langer Eiskeil an der Nordwand der Eversschen Kiesgrube am Nordschacht $5 \mathrm{~km}$ westl. Helmstedt (Nr. 10, Karte 1). Reste einer Grundmoräne noch am Top vorhanden. Erläuterung im Text. Foto Sommer 1966

Fig. 13: Mainly gravel filled ice-wedge cast with a length of $5 \mathrm{~m}$ at the northern wall of the gravel pit Evers at the Nordschacht $5 \mathrm{~km}$ west of Helmstedt (nr. 10, fig. 1). The structure is polygenetic as can be derived from the dark morainic filling material (Drenthe or Elster moraine) in the top of the ice-wedge cast The formerly existant Weichelian loess was removed. Photo summer 1996.

lösse" mit dunklen Sandlagen (wahrscheinlich aus der näheren Umgebung stammend) abwechseln. Bei den in der ehemaligen Ziegeleigrube Einbeck gefundenen Formen handelt es sich um jungweichselzeitliche Phänomene; denn sie wurden in dem mit 8-10 m Dicke mächtigsten und vollständigsten Jungweichsellößprofil gefunden, das in Niedersachsen je gefunden wurde (vgl. Brosche \& Walther 1991: 6 ff.).

\section{Löß-Flugsand-Bildungen}

Während der über 30 Jahre andauernden Beobachtungen an der nördlichen Lößgrenze im Untersuchungsgebiet, die von Helmstedt nach Braun-

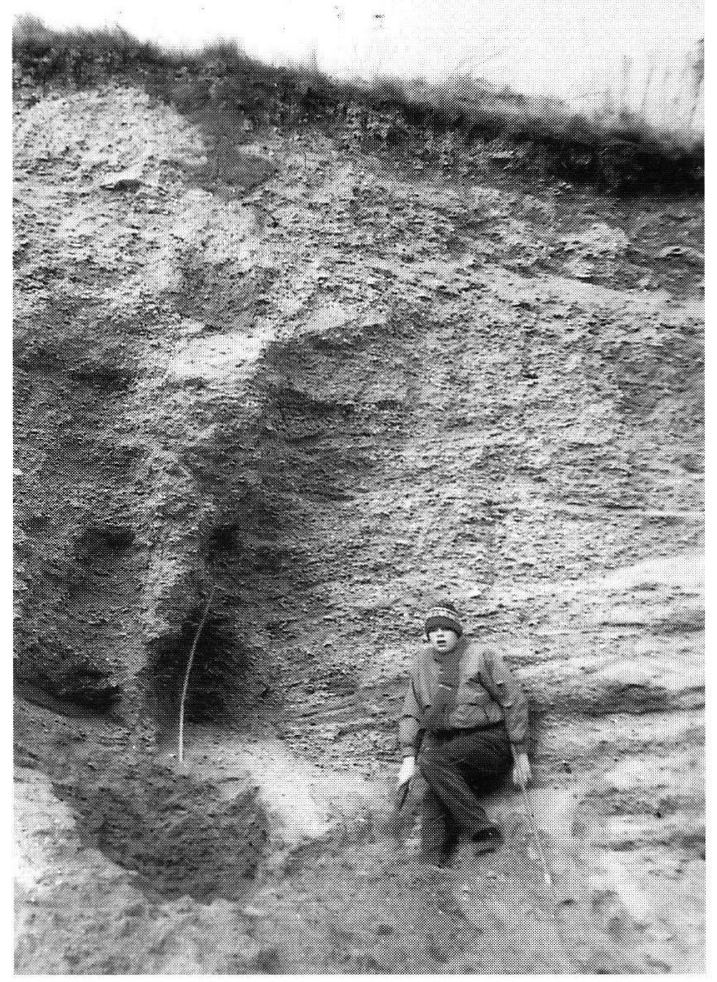

Abb. 14: Rieseneiskeil von 5,0 bis $5,5 \mathrm{~m}$ Länge in der Gemeindekiesgrube Winnigstedt (Nr. 16, Karte 1). Südlich Winnigstedt an der Straße Winnigstedt - Mattierzoll gelegen. 0,5 - 0,6 m unterhalb des 1,2 m langen Maßstabs endet die Eiskeilpseudomorphose mit einer Spitze. Füllmasse des Eiskeils in den unteren 3,5 Metern viele Kalkschotter führende Kiese und Schotter, im oberen Teil Kiese mit Löß, der überwiegend auf dem Geländerücken abgetragen ist. Foto Februar 1994.

Fig. 14: Very long ice-wedge cast with a length of $5.0-5.5 \mathrm{~m}$ in the gravel pit of Winnigstedt (south of Schöppenstedt - nr. 16, fig. 1). Scale has a length of $1.2 \mathrm{~m} .0 .5-0.6 \mathrm{~m}$ downwards of the scale, the ice-wedge ends with a split. The lower $3.5 \mathrm{~m}$ of the structure are filled with gravel that correspondends with the gravel of the whole pit. The upper parts are filled by a mixture of loess and gravel, the uppest part contains Weichselian loess This feature also is of a polygenetic origin. Photo February 1994.

schweig bis Hannover zieht, wurde besonders darauf geachtet, ob ein allmählicher Übergang von einer Flugsandfazies über eine Löß-Flugsand-Fazies zu einer reinen Lößfazies zu beobachten ist. Das Hauptbeobachtungsergebnis lautet, daß der Löß als gelber, im unteren Teil bei ausreichender Mächtigkeit kalkhaltiger Fernlöß abrupt einsetzt. Das geschieht z. B. in Form von etwa $1 \mathrm{~m}$ mächtigen Lößdecken im Gebiet von Süpplingen (westl. Helm- 


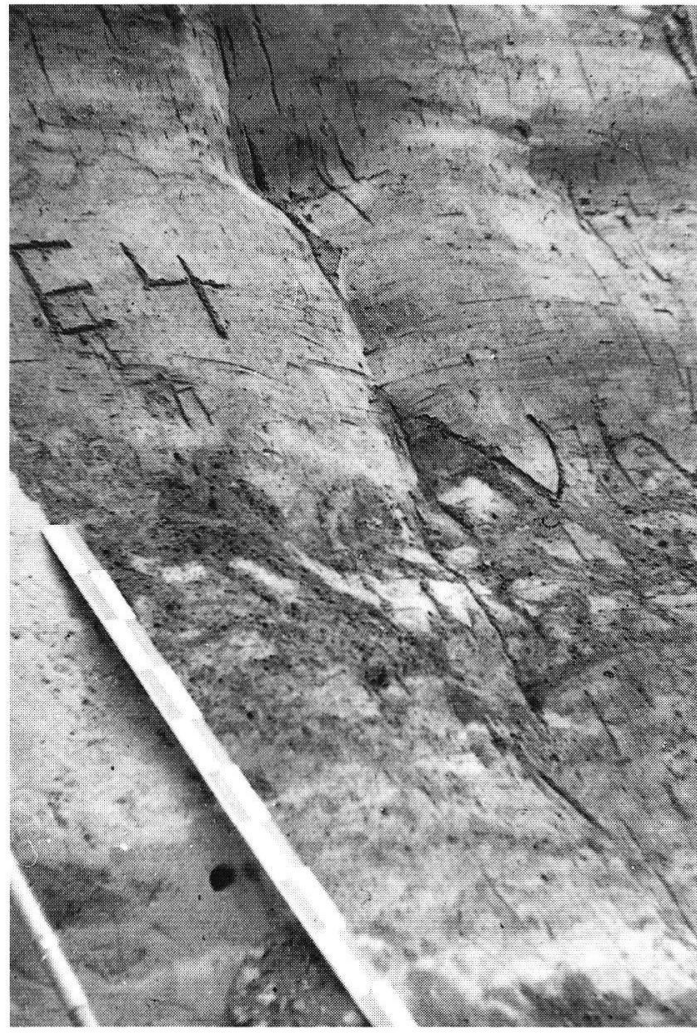

Abb. 15: Kleinkeile am Unterrand des E 4-Naßbodens, de in der niedersächsisch-nordhessischen Nomenklatur dem wj $3 \gamma$-Naßboden entspricht. Maßstab $1 \mathrm{~m}$. Foto Sommer 1977. Ehemalige Ziegeleigrube Sudhoff bei Einbeck

Fig 15: "Kleinkeile", that means little ice-wedge casts with a length of $0.2 \mathrm{~m}$ at the lower ridge of the $\mathrm{E}$ 4-Narboden accord ing to the nomenclature of Schönhals, Rohdenburg \& Semmel (1964). Pit of the former brick factory Sudhoff in Einbeck (southern part of Lower Saxony). Scale $1 \mathrm{~m}$. A permafrost soil cannot be derived from this features. Photo summer 1977.

stedt). In Dellen kann der Löß bereits $1 \mathrm{~km}$ südlich der nördlichen Lößgrenze in der Kiesgrube Evers am Nordschacht 3-4 m Dicke erreichen. Nur in einem Falle, der durch die Abb. 16 (Nr. 2, Abb. 1) dokumentiert wird, ließ sich eine deutliche Abfolge von 0,6-0,8 m Löß im Top und einer Löß-Flugsand-Sequenz beobachten, wie sie $\mathrm{m}$.W. bisher nirgends abgebildet oder beschrieben wurde. Die hellen und weißgelben Bänder dokumentieren den im tieferen Untergrund und nördlich der nördlichen Lößgrenze anstehenden eozänen terrestrisch entstandenen Sand der nördlichen Helmstedter Mulde. Dieser mehrfache, im unteren Teil des Aufschlusses zu beobachtende Sedimentwechsel ist in der Weise zu deuten, daß anfangs höhere Windgeschwindigkeiten und/oder von $\mathrm{N}$ kommende Winde eine bedeutende Rolle gespielt haben müssen, während im weiteren Verlauf der äolischen Prozesse der Ferntransport von typischem feinem gelbem Löß vorge-

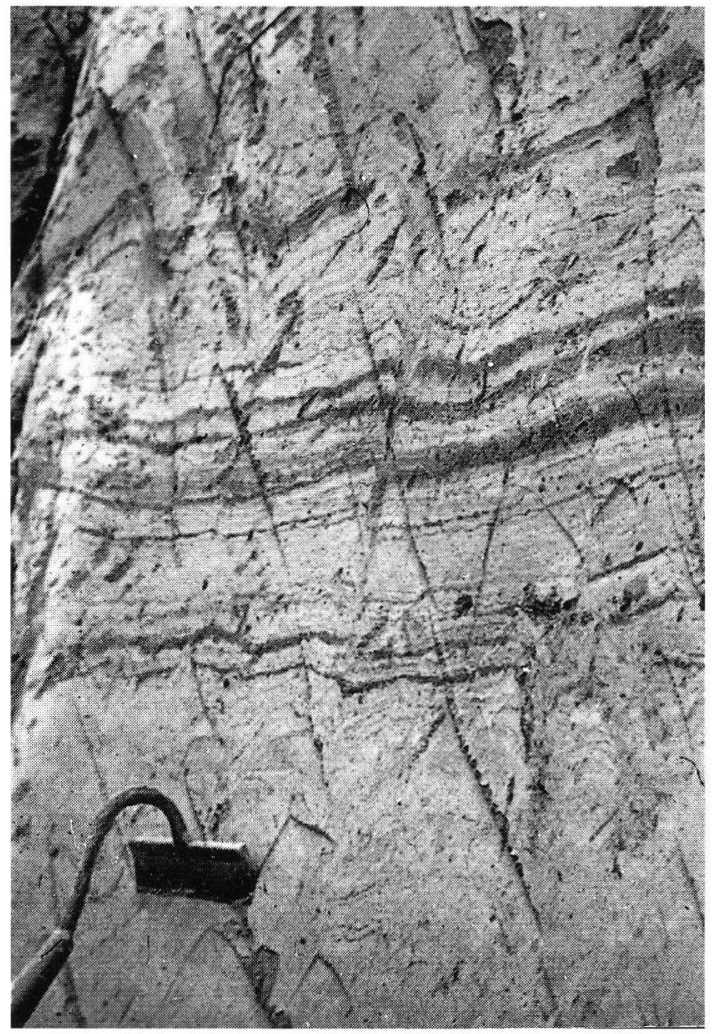

Abb. 16 : Kissenboden und syngenetische Kleinkeile im Bereich des wj $4 \alpha$-Lösses in der ehemaligen Ziegeleigrube Sudhoff bei Einbeck. Maßstab : Breite des Schabers ca. 15 $\mathrm{cm}$. Foto Sommer 1977.

Fig. 16: Same locality as Fig. 15. So called "Kissenboden" and syngenetic mini ice-wedge casts in Weichselian loessic deposits as in Fig 15. Wj $4 \alpha$-loess according to the terminology of Rohdenburg \& Meyer (1966) in the southern part of the pit. Scale $15 \mathrm{~cm}$ broad. Photo summer 1977.

herrscht haben muß. Wechselfolgen von echtem äolischem gelbem Löß, der auf Ferntransport hindeutet, und lokalem gröberem Material (hier mittelund unteroligozänen äolischen Sanden) innerhalb mächtiger Jungweichsellößfolgen wurden auch häufig in den Tagebauen Treue und Helmstedt gefunden.

\section{Asymmetrische Täler}

Nur in zwei Fällen wurden klimatisch bedingte, sekundär-asymmetrische Täler i. S. Posers \& Müllers (1951) innerhalb des Untersuchungsgebietes angetroffen. Es handelt sich um die mehrere Kilometer $\mathrm{S}-\mathrm{N}$ ziehenden Täler westl. der Lübbensteine (westl. Helmstedt - Nr. 3 a, Abb. 1) und um das bekanntere Schuntertal in seinem Abschnitt zwischen Frellstedt, Süpplingen und Süpplingenburg (Abb. 18, Nr. 3 b, Abb. 1). Substratunterschiede fallen hier als Er- 


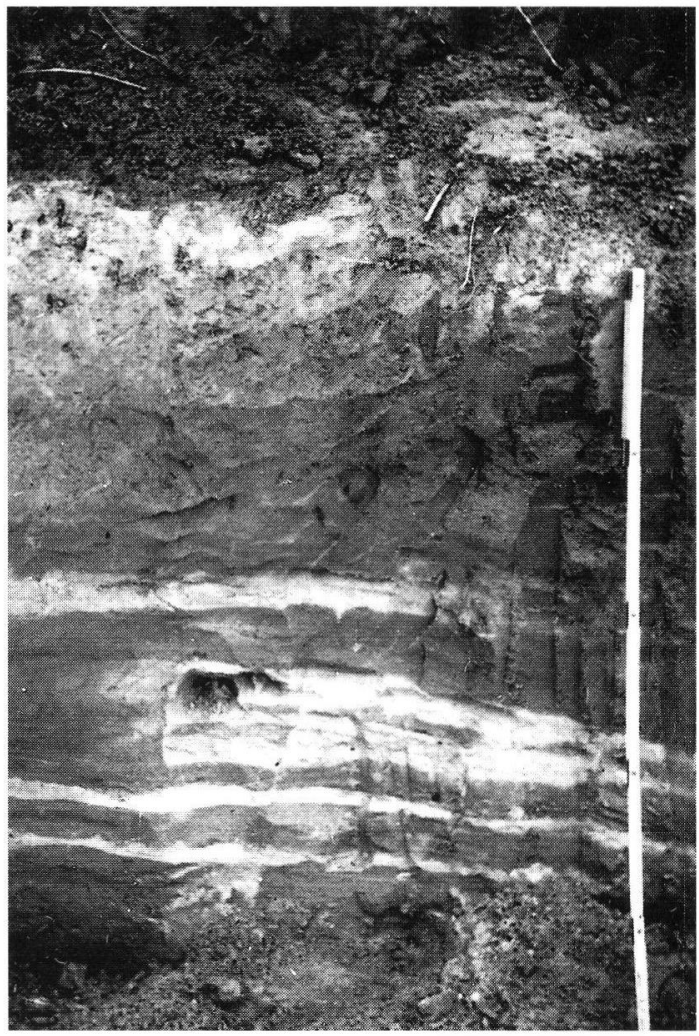

Abb. 17: Sequenz von gelb-orangefarbenem Löß und von Bändern eozänen weißen Sandes in der Heinrich-KrempStraße (Stadtgebiet von Helmstedt). Maßstab $1 \mathrm{~m}$. Über Maßstab rezenter Boden. Foto Sommer 1965

Fig. 17: Layer-sequence of yellow orange coloured loess and white Eozenic sand in the Heinrich-Kremp-Straße (Helmstedt). Neighbourhood of the Northern loess border in Lower Saxony. Scale $1 \mathrm{~m}$; recent soil above the scale. Photo summer 1965.

klärungsursache aus. Das Asymmetrieverhältnis beträgt bei einem Neigungswinkel von 20-25 Grad (EHang) und 3-4 Grad (W-Hang) 5:1 bis 6:1, d. h. das Schuntertal schnitt sich seit dem Drenthe-Stadium, deutlich nach E wandernd, allmählich $15-20 \mathrm{~m}$ in die hier weit verbreiteten quartären Platten ein und schälte sozusagen die quartären Sedimente von dem älteren Untergrund (eozäne Sande und lockere Keupergesteine) im Rahmen seiner Ostverlagerung ab.

\section{Zusammenfassung und Schlußfolgerung}

Im zweiten Teil des gleichnamigen Aufsatzes wird mit Hilfe eines reichhaltigen Bildmaterials der recht vielfältige Formenschatz der einzigen echten Dauerfrostbodenanzeiger des Untersuchungsgebietes, der Eiskeile, vorgestellt. Im ersten Teil (Kap. 2) werden saalezeitliche oder vielleicht noch ältere Eiskeilspaltennetze dokumentiert, die in Ahlum (Nr. 18, Abb. 1) Tiefen von $6 \mathrm{~m}$ erreichen und sich aufgrund ihres Füllmaterials und der Deckschichten recht gut datie- ren lassen. In Barmke (Nr. 12, Abb. 1) wurden neben normalen Eiskeilpseudomorphosen mehrere mindestens saalezeitliche Eisgänge, Eisadern und Eisblätter beschrieben. - Die einphasig entstandenen Eiskeile des Untersuchungsraumes mit Längen von 2,0 bis 2,5 Metern sind nach Beurteilung des Füllund Deckmaterials weichselzeitliche Bildungen (Kap. 3). - Einen weiteren Leckerbissen unter dem periglazialen Formeninventar des Untersuchungsgebietes stellen im Kap. 4 behandelte mehrphasig entstandene Eiskeilpseudomorphosen dar, die entweder nur in einer Eiszeit (wahrscheinlich in der Saaleeiszeit) zwei Eiskeilbildungsphasen erfuhren (Nr. 10, Abb. 1) oder eine saaleeiszeitliche und weichseleiszeitliche Keilbildung in demselben Eiskeil anzeigen (Nr. 16, Abb. 1). In Kap. 5 werden einige Beispiele von innerweichselzeitlichen, bis $20 \mathrm{~cm}$ tiefen „Kleinkeilen“ und Kissenbodenstrukturen behandelt, die beide keinen Dauerfrostboden voraussetzen, sondern temporären, nicht sehr tief reichenden Frostboden. Vom Nordrand der nördlichen Lößgrenze werden als Sonderbeispiel Löß-FlugsandFolgen bekannt gemacht, die in dieser Form selten bzw. überhaupt noch nicht beobachtet wurden. Der Abschluß des Dokumentarteils ist einigen sekundär-asymmetrischen Tälern aus dem Raum westlich Helmstedt gewidmet (Nr. 3 a und 3 b, Abb. 1). Anders als in den durchweg quartären Sedimenten des nördlichen Alpenvorlandes (z. B. POSER \& MülLER 1951; KARRASCH 1970) und in anderen, überwiegend aus quartärem Lockermaterial bestehenden Gebieten stellen in unserem Untersuchungsraum klimabedingte asymmetrische Täler seltene Ausnahmen dar. Während in Teil 1 des gleichnamigen Aufsatzes (Eiszeitalter und Gegenwart, Bd. 46) hauptsächlich Aussagen zur Tiefe des saale- und weichselzeitlichen Auftaubodens gemacht wurden, die sich mit den Ergebnissen POSERS (1947 ff. - zusammenfassend nachzulesen bei WashBuRn 1979, S. 285 ff.) weitgehend decken, sind die in diesem Beitrag gemachten Beobachtungen eher dazu geeignet, mittlere und maximale Eiskeiltiefen für verschiedene Phasen festzulegen. Insgesamt gesehen scheinen die längsten Eiskeile (von 5,5 bis $6 \mathrm{~m}$ Länge) auf die Saalekaltzeit oder die Elsterkaltzeit beschränkt zu sein. Aus diesem Zeitraum stammen auch die einzigen syngenetischen bzw. synchronen Eiskeile (Oker-Mittelterrasse nördl. Isingerode - Nr. 23, Abb. 1) und mehrere Eiskeilspaltennetze. - Fast alle weichselzeitlichen Eiskeile wurden nicht länger als $2 \mathrm{~m}$. Nur in einem Falle erreichte ein mehrphasig entstandenes weichselzeitliches Gebilde in einem vollständigen weichselzeitichen Lößprofil eine Länge von knapp 5 Metern (Brosche \& Walther 1991, S. 26, Abb. 9).

$\mathrm{Zu}$ dem von Look (1967) angeblich entdeckten Pingo im Ostelm nördlich von Eitzum vermag ich keine abschließende Aussage zu machen. KARTE \& LIEDTKE 


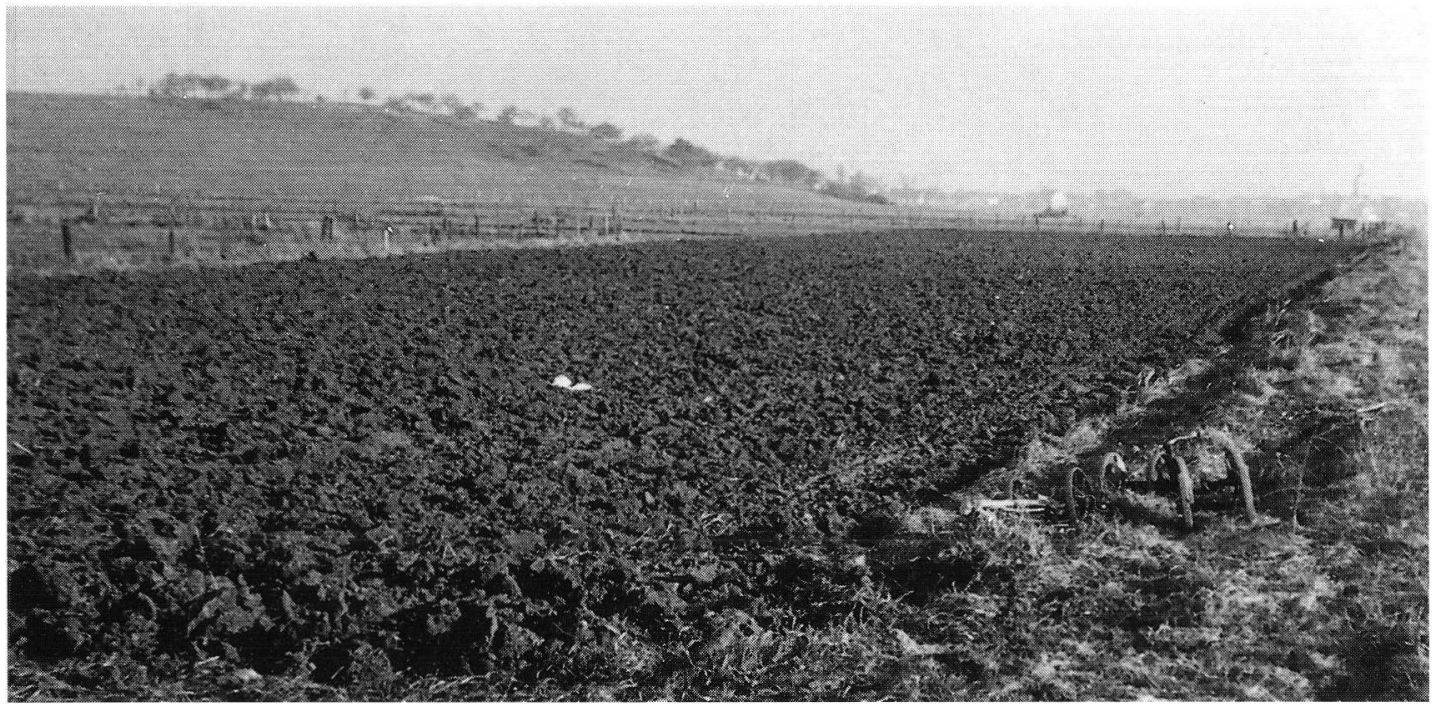

Abb. 18: Sekundär-asymmetrisches Schuntertal südöstl. Süpplingen (Nr. 3b, Karte 1). Blick in südliche bis südöstliche Richtung. Der Steilhang mit maximalen Neigungen von $20-25^{\circ}$ besteht im unteren Teil aus eozänen Sanden (links) bzw. Mergeln des Mittleren Keupers (rechts). Im oberen Teil stehen glazifluviale (wahrscheinlich drenthezeitliche) Sedimente an, die wie in der benachbarten Eversschen Kiesgrube von Grundmoräne und weichselzeitlichem Löß bedeckt sind. Foto Sommer 1966.

Fig. 18: Climatic asymmetric valley of the Schunter river north of Frellstedt (southwest of Helmstedt - nr. 3b, fig. 1). The observer looks from Northwest to Southeast in the valley, which extends here from South to North. The steel slope shows an inclination of $20-25^{\circ}$. The slope consists of Eocene sand (in the left part) and Keuper marl (in the right part) at the bottom and probably Saalian material and Weichselian loess in the top. Photo summer 1965.

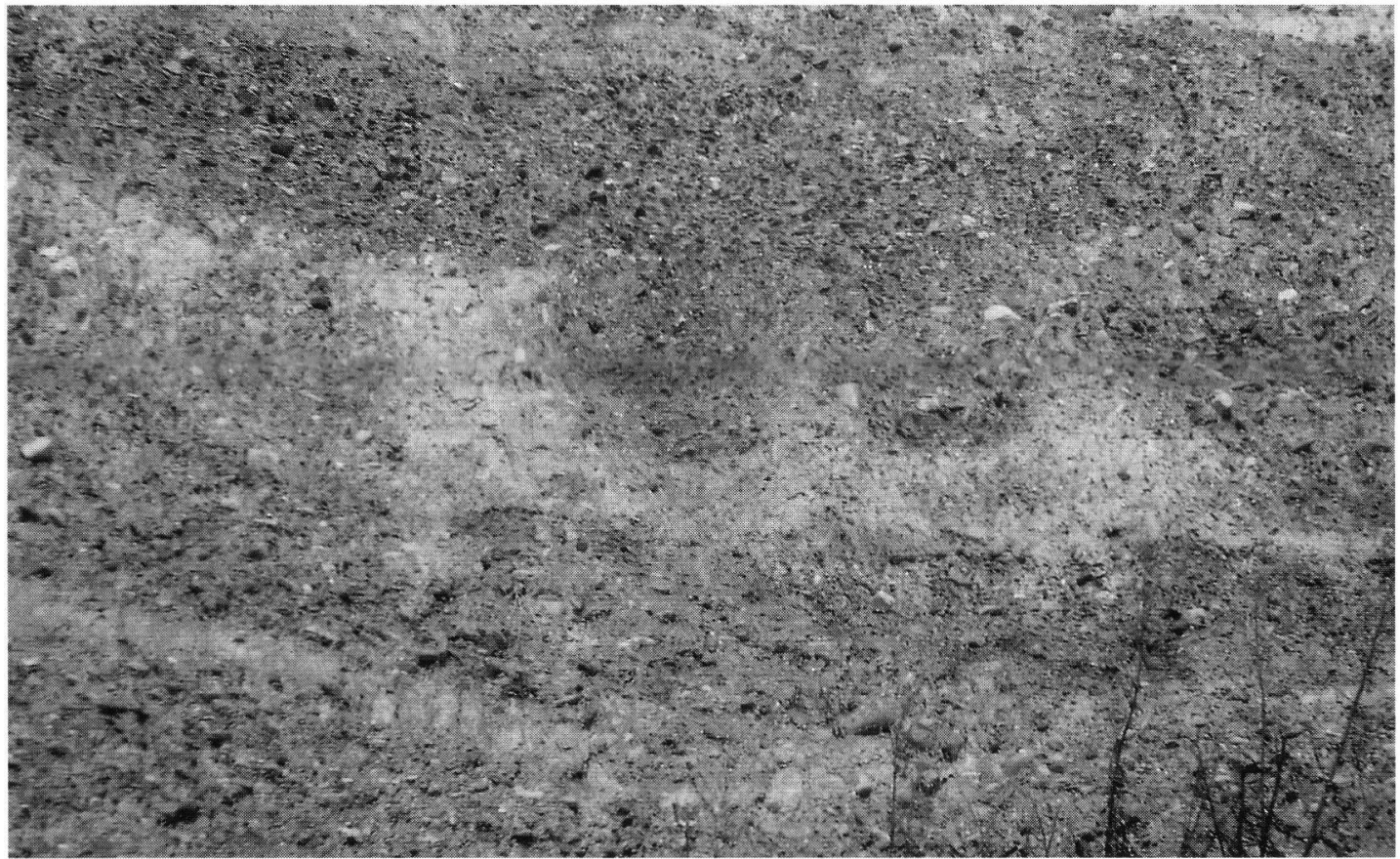

Abb. 19: Syngenetische Kryoturbationsformen (Taschen- bzw. Wannenböden) in der Oker-Mittelterrasse nördl. Isingerode (südl. Wolfenbüttel). Ehemals vorhandener Würm-Löß im Hangenden künstlich entfernt. Schluffige Zwischenlagen begünstigten die Entstehung der frostdynamischen Strukturen. Wandhöhe ca. 3 m-3,5 m. Foto Februar 1995.

Fig. 19: Syngenetic cryoturbation forms ("Taschen"- or "Wannenböden") in the middle terrace of the Oker river (south of Wolfenbüttel). Würmian loess existing at the top formerly has been put away by men. Silty layers favoured the formation of the cryoturbation pakets. Hight of the exposure $3-3.5 \mathrm{~m}$. Photo. February 1995. 
(1979 - in WASHBURN 1979, S. 300 abgedruckt) sind offenbar nicht gewillt, diese Form als echten Pingo anzuerkennen.

Während die Eiskeile als echte Dauerfrostbodenindikatoren anzusehen sind, trifft dies auch nach meiner Auffassung nur für die Kryoturbationsformen (Würgeböden in mehr oder weniger homogenem Schottermaterial am Weddebach nördl. des Harliberges bei Vienenburg und in der Oker-Mittelterrasse bei Isingerode - Teil 1 in Bd. 46 von E. u. G.) zu, die erhebliche vertikale Ausmaße von 1,0 bis $1,5 \mathrm{~m}$ aufweisen, im letzteren Fall sogar deutlich syngenetische Formen i. S. von GaLLwitz (1949) bzw. synchrone Formen i. S. von KaISER (1960) darstellen. Für die sekundär-asymmetrischen Täler besteht schon seit langem Einigkeit darüber, daß sie keine strengen Dauerfrostbodenanzeiger sind.

Während man bis 1960 und danach noch annahm, daß ein geschlossener Dauerfrostboden Jahresdurchschnittstemperaturen von wenigstens $-2^{\circ}$ voraussetze (Kaiser 1960: 135) und unter diesen Umständen aktive Eiskeilbildung möglich sei, herrscht heute die Erkenntnis vor, daß Eiskeile zu ihrer Entstehung eine Jahresdurchschnittstemperatur von mindestens $-6^{\circ}$ (Semmel 1975:18) benötigen. Unter Anwendung dieser Erkenntnisse läßt sich für den Untersuchungsraum, in dem heute Jahresmitteltemperaturen von $8-9^{\circ}$ herrschen, schließen, daß während der kältesten Abschnitte der Saale- und Weichseleiszeit (vielleicht auch schon in der Elstereiszeit), in denen die Eiskeile und Eiskeilnetze entstanden sind, eine Temperaturdepression von mindestens 14 bis 15 Grad anzusetzen ist.

\section{Dank}

Herr Priv.-Doz. Dr. M. Walther, Inst. f. Geogr. Wiss. der FU Berlin, Fachrichtung Physische Geographie, führte mit mir gemeinsam viele Untersuchungen in den Tagebauen der Braunschweigische Kohlebergwerke, Helmstedt (BKB), durch. Dafür gilt ihm mein Dank.

\section{Schriftenverzeichnis}

Bartels, G. \& Rohdenburg, H. (1968): Fossile Böden und Eiskeilhorizonte in der Ziegeleigrube Breinum und ihre Auswertung für die Reliefentwicklung im Jungquartär. - Göttingen Bodenkundliche Berichte, 6: 109 - 126; Göttingen.

Brosche, K.-U. (1964): Über Periglazialerscheinungen im Lappwald und in seinem nächsten Vorland. - Seminararbeit Universität Göttingen, angefertigt bei Prof. Dr. H. Poser, unveröffentlicht; 80 S.; Göttingen.

- (1968): Struktur- und Skulpturformen im nördlichen und nordwestlichen Harzvorland. - Göttinger Geographische Abhandlungen, 45: 236 S., 2 Textabb., 10 Beilagen; Göttingen.

- (1969: Über die Beziehungen von Rumpfflächen zu Schichtkämmen und Schichtstufen sowie Beobachtungen an einigen wichtigen Strukturformtypen, erläutert an Beispielen aus dem nördlichen und nordwestlichen Harzvorland. - Zeitschr. f. Geomorph., 13: 207 - 216; Berlin.

- (1996): Wirkungen des pleistozänen kaltzeitlichen Klimas, insbesondere des Bodenfrostes, in den Sedimenten des östlichen Ostfalen (Raum Hannover - Wolfsburg - Helmstedt - Bad Harzburg - Salzgitter Bad - Hannover) Teil 1. Eiszeitalter und Gegenwart: 46: 1-17. Hannover 1996.

-\& W WLTHER, M. (1978): Die jungpleistozänen Lößdeckschichten der Braunkohlentagebaue der Braunschweigischen Kohlebergwerke (BKB) zwischen Helmstedt und Schöningen. - Eiszeitalter und Gegenwart, 28: 51-67; Öhringen.

- \& WaLTHER, M. (1991): Untersuchungen zur Lößstratigraphie und jungpleistozänen Formung in Südniedersachen und im östlichen Westfalen. - Geographica -- Oekologica. - Arbeiten zur Geographie und Ökologie, 2: 52 S., 13 Abb., 2 Karten, 7 Fotos auf 3 Tafeln; Berlin.

BrüNING, H. (1956): Fossile Frostbodenerscheinungen aus dem Stadtgebiet von Magdeburg und aus der Magdeburger Börde. Hallesches Jahrbuch für Mitteldeutsche Erdgeschichte, 2, 3; Halle.

- (1957): Eiszeitliche „Klein-Frostbodenformen“ im Talrandlöß von Magdeburg. - Hallesches Jahrbuch für Mitteldeutsche Erdgeschichte, 2 , 4: Halle.

- (1958): Museumsarbeit in den Baugruben einer Großstadt. Der Präparator, Zeitschrift für Museumstechnik, 4.

- (1959): Periglazialerscheinungen und Landschaftsgenese im Bereich des mittleren Elbetales bei Magdeburg. - Studien über die Periglazialerscheinungen in Mitteleuropa, herausgegeben von Hans Poser, Göttinger Geographische Abhandlungen, 6 Abb., 18 Fig. als Beilagen, 80 S.; Göttingen.

- (1966): Vorkommen und Entwicklungsrhythmus oberpleistozäner Periglazial-Erscheinungen und ihr Wert für pleistozäne Hangformung. - Forschg. z. Dtsch. Landeskunde 156, 94 S. und $52 \mathrm{Abb}$., Bad Godesberg.

GofDecke, R. (1966): Die Oberflächenformen des Elm. - Göttinger Geogr. Abhandlungen, 35; Göttingen.

Hempel, L. (1955): Frostbodenbildung und Lößanwehung in der Würmeiszeit auf Muschelkalk und Buntsandstein bei Göttingen. - Abhandlungen der Akademie d. Wiss. u. d. Lit. in Mainz. Math.-naturwiss. Kl., Jhg. 1955, Nr. 2; Mainz.

Herrmann, R. (1929): Erdgeschichtliche Grundfragen der Oberflächenformung in Mitteldeutschland. Festschrift des 23. Deutschen Geographentages in Magdeburg, hrsg. von O. SCHLÜTER \& E. Buume : 71 - 108; Braunschweig / Berlin / Hamburg.

KAISER, K. (1958): Wirkungen des pleistozänen Bodenfrostes in den Sedimenten der Niederrheinischen Bucht. - Eiszeitalter und Gegenwart, 9: 110 - 129, Öhringen.

- (1960): Klimazeugen des periglazialen Dauerfrostbodens in Mittel- und Westeuropa. - Eiszeitalter und Gegenwart, 11: 121 - 141, Öhringen (Rau).

- (1966): Das Quartär zwischen Harz und Elbe. - Eiszeitalter und Gegenwart, 17: 200 - 204; Öhringen.

KeIlнAск, K. (1931): Über „atektonische Faltung“ (Stiche) in der Trias des Harzvorlandes und über einen merkwürdigen Fall des Hakenschlagens. - Zeitschr. d. Dtsch. Geol. Ges., 83.

LIETKE, H. (1975): Die nordischen Vereisungen in Mitteleuropa. Forschungen zur Deutschen Landeskunde, 204; Bonn - Bad Godesberg.

LOOK, E. R. (1968): Geologisch-stratigraphische Untersuchungen in Sedimenten der Elster- und Saale-Eiszeit (Pleistozän) am Elm, östlich Braunschweig. Mitteilungen aus dem Geologischen Institut der Technischen Hochschule Hannover, 6: 108 S., 18 Abb., 4 Tab., 27 Taf.; Hannover.

- (1984): Geologie und Bergbau im Braunschweiger Land. Berichte der Naturhistorischen Ges. Hannover, 127: 1 - 467; Hannover.

LuDwIG, A. (1958): Beobachtungen im Pleistozän des nordöstlichen Harzvorlandes. - Geologie, 7: 769 - 793; Berlin.

Mania, D. u. Toeprer, V. (1973): Königsaue, Gliederung, Ökologie und mittelpaläolithische Funde der letzten Eiszeit. - Veröffentlichungen des Landesmuseums f. Vorgeschichte Halle, 26: 164 S.; Halle.

MANIA, D. (1965): Stratigraphie, Genese und Palökologie des Quartärs in der Ascherslebener Depression und ihrer Umgebung. Diss. Halle.

- (1967): Das Jungquartär aus dem ehemaligen Ascherslebener See im Nordharzvorland. - Petermanns Geographische Mitteilungen, 111: 257 - 273; Gotha. 
- \& Stechemesser, H. (1970): Jungpleistozäne Klimazyklen im Harzvorland. - Petermanns Geographische Mitteilungen, Erg.Heft, 274: 39 - 55; Gotha.

MERKT, J. (1968): Erläuterungen zur Karte der Lößverbreitung in Südniedersachsen. - Geol. Jahrbuch, 86: 107 - 112; Hannover.

Poser, H. (1947 a): Dauerfrostboden und Temperaturverhältnisse während der Würmeiszeit im nicht vereisten Mittel- und Westeuropa. - Naturwissenschaften, 34: 10 - 18, Berlin.

- (1947 b): Auftautiefe und Frostzerrung im Boden Mitteleuropas während der Würm-Eiszeit. - Naturwissenschaften, 34: 262 - 267 und 323 - 328; Berlin.

- (1948 a): Boden- und Klimaverhältnisse in Mittel- und Westeuropa während der Würm-Eiszeit. - Erdkunde, 2: 53 - 68; Bonn.

- (1948 b): Aolische Ablagerungen und Klima des Spätglazials in Mittel- und Westeuropa. - Naturwissenschaften, 9: 269 - 275 und $307-312$; Berlin.

- (1951): Die nördliche Lößgrenze in Mitteleuropa und das spätglaziale Klima. Eiszeitalter und Gegenwart, 1: 27 - 55; Öhringen (Rau).

Rohdendurg, H. (1965): Die Muschelkalk-Schichtstufe am Ostrand des Sollings und Bramwaldes. - Göttinger Geogr. Abh., 33; Göttingen.

- (1966): Eiskeilhorizonte in südniedersächsischen und nordhessischen Lößprofilen. - Mitteilungen d. Deutschen Bodenkundl. Ges., 5: 137 - 170; Göttingen.

- (1968): Jungpleistozäne Hangformung in Mitteleuropa. Beiträge zur Kenntnis, Deutung und Bedeutung ihrer räumlichen und zeitlichen Differenzierung. - Göttingen Bodenkundl. Berichte, 6: 3 - 107; Göttingen.
- \& Meyer, B. (1966): Zur Feinstratigraphie und Paläopedologie des Jungpleistozäns nach Untersuchungen an südniedersächsischen und nordhessischen Lößprofilen. - Mitteilungen der Deutschen Bodenkundl. Gesellschaft, 5: 5 - 131; Göttingen.

Schönhals, E., Semmel, A. \& H. Rohdenburg (1964): Ergebnisse neuerer Untersuchungen zur Würmlöß-Gliederung in Hessen. - Eiszeitalter und Gegenwart, 15: 199 - 206; Öhringen.

Semmer, A. (1985): Periglazialmorphologie. - Erträge der Forschung. Bd. 231. Wiss. Buchgesellschaft Darmstadt. 50 Abb. im Text. Darmstadt.

Trombotto, D. \& Ahumada, A.-L. (1995): Die Auswirkung alter Kryomere auf die „Rodados Patagonicos“ in Nordpatagonien, Argentinien. - Eiszeitalter und Gegenwart. Bd. 45: 93 - 108. Stuttgart.

WALTHER, M. (1979): Verbreitung und Stratigraphie letzteiszeitlicher Lößfolgen in der norddeutschen Lößzone südlich der nördlichen Verbreitungsgrenze bis zu den Mittelgebirgen hin (Nordrhein-Westfalen, Südniedersachsen). - Schriftliche Staatsexamensarbeit, Wiss. Landesprüfungsamt Berlin; Berlin.

WALTHER, M. \& BROSCHE, M. (1983): Zur Bedeutung der Lößstratigraphie für die Rekonstruktion des jungpleistozänen Klimas im nördlichen Mitteleuropa am Beispiel norddeutscher Lößprofile. - Ber. naturhist. Ges. Hannover, 125: 97 - 159: Hannover.

WASHBURN, a. L. (1979): Geocryology. - A survey of periglacial processes and environments. $406 \mathrm{~S}$. London.

WOLDSTEDT, P. \& DUPHORN, K. (1974): Norddeutschland und angrenzende Gebiete im Eiszeitalter; Stuttgart.

Manuskript eingegangen am 29.08. 1996 